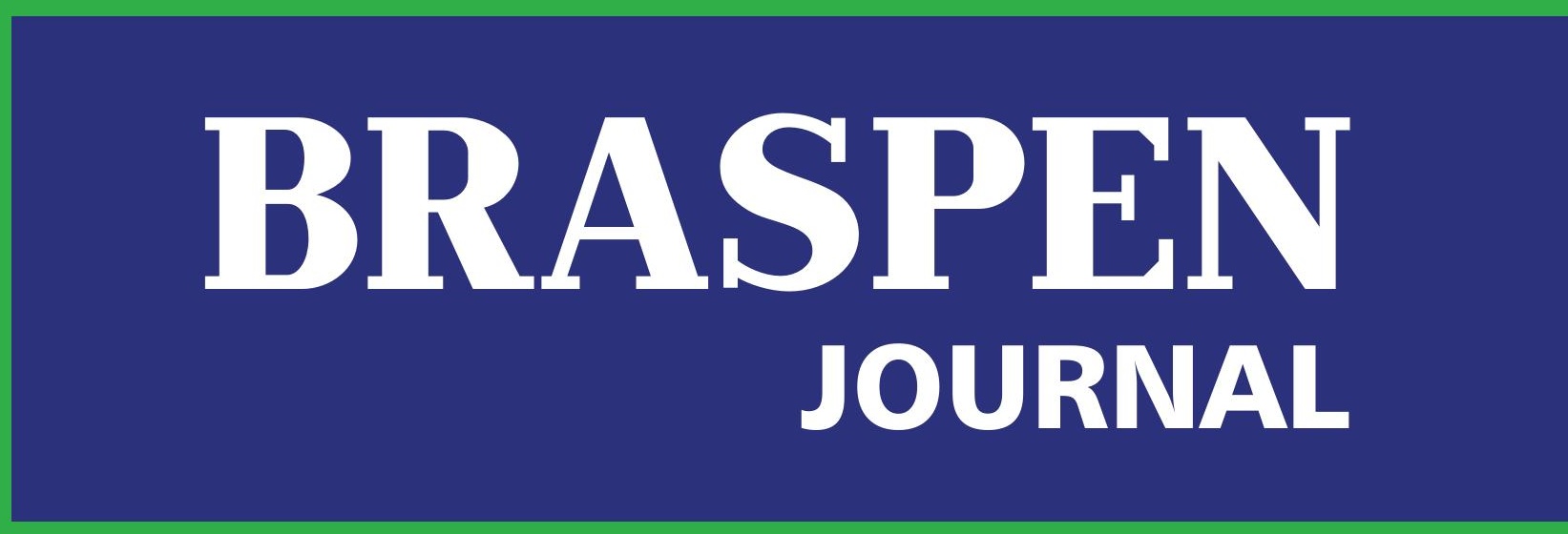

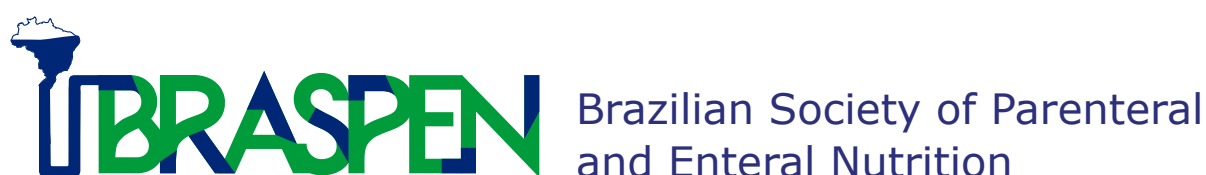 SBNPE
}

\section{Campanha Diga Não à Lesão por Pressão}

Liane Brescovici Nunes de Matos, Silvia Maria Fraga Piovacari, Ricardo Ferrer, Juliana Tepedino Martins Alves,

Thaisa Assis, Amanda C. M. A. G. Brandão, Melina Gouveia Castro, Guilherme Duprat Ceniccola,

Haroldo Falcão Ramos da Cunha, Maria Carolina Gonçalves Dias, Rogério Dib, Diana Borges Dock-Nascimento, Maria Emília Gaspar Ferreira, Ivens Willians Silva Giacomassi, Rodrigo Costa Gonçalves, Thiago José Martins Gonçalves, Claudia Satiko Takemura Matsuba, Julieta Regina Moraes, Mara Rubia de Moura, Priscila Barsanti de Paula Nogueira,

Bruna Fernanda Camargo Silva Parra, Paula Pexe-Machado, Ricardo Tadeu Prete, Fernanda Antunes Ribeiro,

Renata Alves dos Santos, Glaucia Fernanda Corrêa Gaetano Santos, Letícia Faria Serpa, Mariana Fernandes Cremasco

de Souza, Diogo Oliveira Toledo, Clarissa Martins Zambelli, Adriano Antonio Mehl, Antonio Carlos Ligocki Campos 



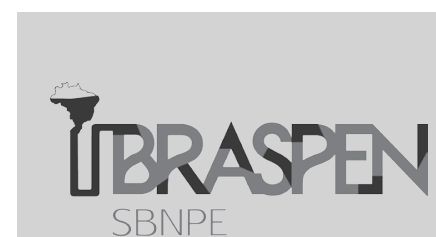

Brazilian Society of Parenteral and Enteral Nutrition

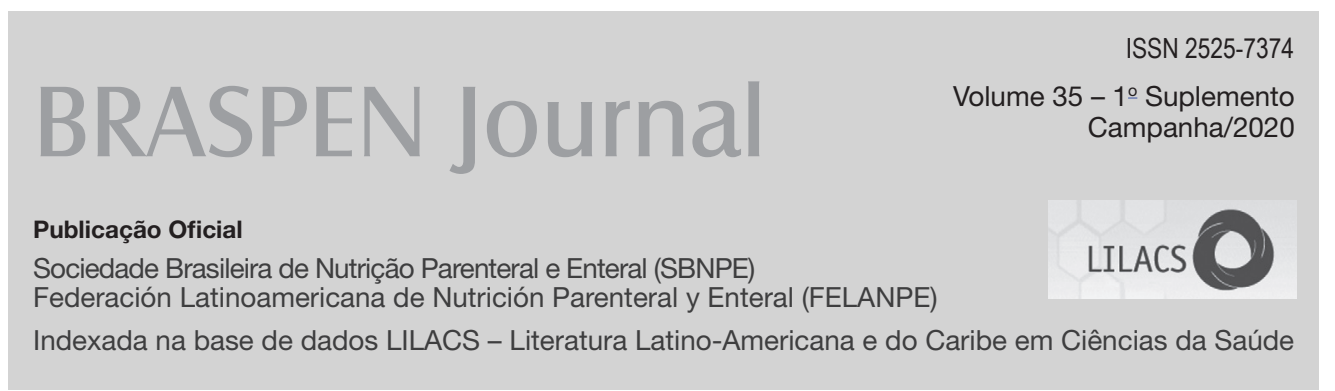

ISSN 2525-7374

- 1o Suplemento Campanha/2020

Publicação Oficia

Sociedade Brasileira de Nutrição Parenteral e Entera (SBNPE)

Indexada na base de dados LILACS - Literatura Latino-Americana e do Caribe em Ciências da Saúde

\section{Editor Chefe:}

José Eduardo de Aguilar-Nascimento

Diretor do Curso de medicina, UNIVAG (Várzea Grande, MT, Brasil)

\section{Editora Executiva:}

Paula Pexe Alves Machado

Centro Universitário de Várzea Grande, Cursos de Medicina e Nutrição.

(Cuiabá/Várzea Grande, MT, Brasil)

\section{MEMBROS DO CONSELHO EDITORIAL:}

\section{Antonio Carlos Ligocki Campos}

Universidade Federal do Paraná, Departamento de Cirurgia do Setor de Ciências da Saúde (Curitiba, PR, Brasil)

Dan Linetzky Waitzberg

Faculdade de Medicina da Universidade de São Paulo, Departamento de Gastroenterologia (São Paulo, SP, Brasil)

Maria Isabel Toulson Davisson Correia

Universidade Federal de Minas Gerais, Departamento de Cirurgia (Belo Horizonte, BH, Brasil)

\section{EDITORES ASSOCIADOS NACIONAIS}

\section{Médicos}

Alessandra Miguel Borges

Centro de Pesquisas Oncológicas de Florianópolis (CEPON)

(Florianópolis, SC, Brasil)

Carlos Antonio Bruno da Silva

Universidade de Fortaleza, Diretoria do Centro de Ciências da Saúde

(Fortaleza, CE, Brasil)

José Raimundo Araujo de Azevedo

Hospital São Domingos, Serviço de Terapia Intensiva (São Luis, MA, Brasil)

Melina Gouveia Castro

Hospital Mario Covas (São Paulo, SP, Brasil)

Odery Ramos Júnior

Universidade Federal do Paraná e Faculdade Evangélica de Medicina do

Paraná, Disciplina de Gastroenterologia (Curitiba, PR, Brasil)

Paulo Roberto Leitão de Vasconcelos

Universidade Federal do Ceará, Faculdade de Medicina (Fortaleza, CE, Brasil)

Roberto Carlos Burini

Universidade Estadual Paulista Júlio de Mesquita Filho, Departamento de Saúde Pública (Botucatu, SP, Brasil)

Roberto José Negrao Nogueira

Universidade Estadual de Campinas, Faculdade de Ciências Médicas (Campinas, SP, Brasil)

\section{Pediatria}

\section{Mario Cicero Falcão}

Faculdade de Medicina da Universidade de São Paulo, Departamento de Pediatria (São Paulo, SP, Brasil)

\section{Rubens Feferbaum}

Faculdade de Medicina da Universidade de São Paulo, Departamento de Pediatria (São Paulo, SP, Brasil)

\section{José Vicente Spolidoro}

Pontifícia Universidade Católica do Rio Grande do Sul, Faculdade de Medicina, Departamento de Pediatria (Porto Alegre, RS, Brasil)

\section{Nutricionistas}

Cristina Martins

Fundação Pró-Renal Brasil (Curitiba, PR, Brasil)

Diana Borges Dock Nascimento

Universidade Federal de Mato Grosso, Departamento de Nutrição (Cuiabá, MT, Brasil)

Graziela Ravacci

Faculdade de Medicina da Universidade de São Paulo, Departamento de Gastroenterologia (São Paulo, SP, Brasil)

Mariana Raslan Paes Barbosa

Faculdade de Medicina da Universidade Federal do Mato Grosso do Sul (Campo Grande, MS, Brasil)

Silvana Paiva Orlandi

Universidade Federal de Pelotas, Faculdade de Nutrição

(Pelotas, RS, Brasil)
Simone Vasconcelos Generoso

Universidade Federal de Minas Gerais, Departamento de Nutrição (Belo Horizonte, MG, Brasil)

\section{Enfermeiros}

Claudia Satiko Takemura Matsuba

Hospital do Coracão-São Paulo, Equipe Multiprofissinal Terapia Nutricional - EMTN (São Paulo, SP, Brasil)

Leticia Faria Serpa

Hospital Alemão Oswaldo Cruz (São Paulo, SP, Brasil)

Maria Isabel Pedreira de Freitas

Universidade Estadual de Campinas, Faculdade de Enfermagem (Campinas, SP, Brasil)

Suely Itsuko Ciosak

Universidade de São Paulo, Departamento de Enfermagem em Saúde Coletiva (São Paulo, SP, Brasil)

\section{Bióloga}

Raquel Susana Matos de Miranda Torrinhas

Faculdade de Medicina da Universidade de São Paulo, Departamento de Gastroenterologia (São Paulo, SP, Brasil)

\section{EDITORES ASSOCIADOS INTERNACIONAIS}

Alessandro Laviano

University of Rome (Roma, Itália)

Andrew Ukleja

Department of Gastroenterology at Cleveland Clinic Florida (Weston, Florida, Estados Unidos)

Carla Prado

University of Alberta (Alberta, Canadá)

Carol Ireton Jones

Nutrition Therapy Specialist, Carrollton, Texas (Carrollton, Texas, Estados Unidos)

Gordon Jensen

University of Vermont College of Medicine (Burlington, Vermont, Estados Unidos)

Nicolas Velasco

Pontifical Catholic University of Chile (Santiago, Chile)

Luiza Kent Smith University of Saskatchewan (Saskatoon, Saskatchewan, Canada)

Paula Alves

Instituto Portugues de Oncologia do Porto de Francisco Gentil (IPOPFG-E.P.E) (Porto, Portugal)

Remy Meier

Medical University Hospital Liestal (Liestal, Switzerland)

Robert Martindale

Oregon Health \& Science University (Eugene, Oregon, Estados Unidos)

Stephen McClave

University of Louisville (Louisville, Kentucky, Estados Unidos)

Vanessa Fuchs

Universidad ANAHUAC and UNAM (Cidade do México, México)

COMITÊ CONSULTIVO

\section{Joel Faintuch}

Faculdade de Medicina da Universidade de São Paulo, Departamento de Gastroenterologia (São Paulo, SP, Brasil)

Steven B. Heymsfield

Pennington Biomedical Research Center (Baton Rouge, Louisiana, Estados Unidos)

Secretária:

Vanice Silva de Oliveira Freitas

Revisora Cientifica:

Rosangela Monteiro

Projeto Gráfico, Diagramação e Revisão:

Criativa Comunicação e Editora

Impressão:

Pontograf 


\section{JBRASPEN SBNPE}

\section{Brazilian Society of Parenteral and Enteral Nutrition}

\section{www.braspen.org}

PRESIDENTE:

Melina Gouveia Castro

VICE-PRESIDENTE

Diogo Oliveira Toledo

\section{PRIMEIRO SECRETÁRIO}

SEGUNDA SECRETÁRIA

Clarissa Martins Saraiva Figueira Zambell

PRIMEIRO TESOUREIRO

Liane Brescovici Nunes de Matos

SEGUNDA TESOUREIRA

Juliana Tepedino Martins Alves

\section{CONSELHO FISCAL}

Paulo César Ribeiro

Silvio José de Lucena Dantas

Suely Itsuko Ciosak

Alberto José de Barros Neto

Lucas Lima de Carvalho

\section{CONSELHO CONSULTIVO}

Ricardo Schilling Rosenfeld

Diana Borges Dock Nascimento

Nara Lucia Andrade Lopes

Karla Lopes Pereira Gomes

Simone Araújo

Marcia de Souza Antunes

Mario Jorge Sobreiro

José Vicente Noronha Spolidoro

COMITÊ DE DEFESA PROFISSIONAL

Fabiano Girade Correa

Maria Carolina Gonçalves Dias

Marcia de Souza Antunes

Jaqueline Almeida Guimarães Barbosa

Ana Maria Furkim

\author{
COMITÊ DE FARMÁCIA \\ Presidente: \\ Wander Campos \\ Vice-Presidente: \\ Michel Kfouri Filho \\ Secretário: \\ Claudete Jacyzen
}

\section{COMITÊ DE NUTRIÇÃO}

Presidente:

Leticia Fuganti Campos

Vice-Presidente:

Guilherme Duprat Ceniccola

Secretária:

Priscila Alves Barreto

\section{COMITÊ DE ENFERMAGEM}

Presidente:

Cláudia Satiko Takemura Matsuba

Vice-Presidente:

Ricardo Tadeu Prete

Secretária:

Fernanda Ramires Tott

\section{COMITÊ DE FONOAUDIOLOGIA}

Presidente:

Maria de Fatima Lago Alvite

Vice-Presidente:

Irene de Pedro Netto

Secretária:

Christiane Lopes de Albuquerque
COMITÊ DA CRIANÇA E DO ADOLESCENTE

Presidente:

Daniela Gomes

Vice-Presidente:

Fernanda Luisa Ceragioli Oliveira

Secretária:

Cláudia Bezerra de Almeida

COMITÊ DE REABILITAÇÃO INTESTINAL

Presidente:

Silvio Dantas

Vice-Presidente:

Maria de Lourdes Teixeira da Silva

Secretário:

João Wilney Franco Filho

COMITÊ DE ASSISTÊNCIA DOMICILIAR

Presidente:

Nara Lucia Andrade Lopes

Vice-Presidente:

Denise Philomene Joseph Van Aanholt

Secretária:

Cristiane D' Almeida

MEMBROS DO COMITÊ EDUCACIONAL

Robson Freitas De Moura

José Eduardo Aguilar Nascimento

Diogo Oliveira Toledo

Letícia Faria Serpa

Ricardo Tadeu Prete

Sérgio Henrique Loss

Ana Paula Noronha Barrere

Silvia Maria Fraga Piovacari

Lindinalda de França Rocha

Décio dos Reis Ribeiro

\section{Sociedade Brasileira de Nutrição Parenteral e Enteral}

BRASPEN Journal, ISSN 2525-7374, é órgão oficial de divulgação da Sociedade Brasileira de Nutrição Parenteral e Enteral (BRASPEN).

Disponível on line: http://www.braspen.org/braspen-journal

Responsabilidade legal: A Sociedade Brasileira de Nutrição Parenteral e Enteral e os editores do BRASPEN Journal não podem ser responsabilizados por erros, danos ou por qualquer consequência de lesão a pacientes ou indivíduos derivados do uso das informações contidas nesta publicação. Os pontos de vista e as opiniões expressas pelos autores não necessariamente refletem aqueles do corpo editorial; tampouco a publicação de anúncios constitui qualquer endosso da Sociedade Brasileira de Nutrição Parenteral e Enteral ou do Corpo Editorial aos produtos anunciados pelos fabricantes.

( 2020 Copyright: Todos os direitos reservados. Os artigos podem ser reproduzidos para uso pessoal. Nenhuma outra modalidade de publicação pode reproduzir os artigos publicados sem a prévia permissão, por escrito, da Sociedade Brasileira de Nutrição Parenteral e Enteral.

Endereço para correspondência: BRASPEN Journal. Rua Abílio Soares, 233 Conjunto 144 - São Paulo, SP, Brasil - CEP: 04005-000 Telefone: (11) 3889-9909 - E-mail: revista@braspen.org 


\title{
CAMPANHA DIGA NÃO À LESÃO POR PRESSÃO
}

\author{
AUTORES \\ Liane Brescovici Nunes de Matos \\ Silvia Maria Fraga Piovacari \\ Ricardo Ferrer \\ Juliana Tepedino Martins Alves \\ Thaisa Assis \\ Amanda C. M. A. G. Brandão \\ Melina Gouveia Castro \\ Guilherme Duprat Ceniccola \\ Haroldo Falcão Ramos da Cunha \\ Maria Carolina Gonçalves Dias \\ Rogério Dib \\ Diana Borges Dock-Nascimento \\ Maria Emília Gaspar Ferreira \\ Ivens Willians Silva Giacomassi \\ Rodrigo Costa Gonçalves \\ Thiago José Martins Gonçalves \\ Claudia Satiko Takemura Matsuba \\ Julieta Regina Moraes \\ Mara Rubia de Moura \\ Priscila Barsanti de Paula Nogueira \\ Bruna Fernanda Camargo Silva Parra \\ Paula Pexe-Machado \\ Ricardo Tadeu Prete \\ Fernanda Antunes Ribeiro \\ Renata Alves dos Santos \\ Glaucia Fernanda Corrêa Gaetano Santos \\ Letícia Faria Serpa \\ Mariana Fernandes Cremasco de Souza \\ Diogo Oliveira Toledo \\ Clarissa Martins Zambelli \\ Adriano Antonio Mehl \\ Antonio Carlos Ligocki Campos
}




\section{Glossário}
LP: $\quad$ lesão por pressão
PU: $\quad$ Pressure ulcer
PCR: $\quad$ proteína C reativa
UTI: $\quad$ unidade de terapia intensiva

EVARUCI: Escala de Avaliação do Risco de Desenvolvimento de Lesão por Pressão em Cuidados Intensivos

ELPO: $\quad$ Escala de Avaliação de Risco para o Desenvolvimento de Lesões Decorrentes do Posicionamento Cirúrgico

EPUAP/

NPIAP/PPPIA: Sistema Internacional de Classificação de Lesões por Pressão

LPTP: $\quad$ lesão por pressão tissular profunda

MUST: $\quad$ Malnutrition Universal Screening Tool

MAN: $\quad$ Mini Nutrition Assessment

NRS 2002: Nutritional Risk Screening 2002

$\mathrm{Cl} \quad \quad$ calorimetria indireta

SNO: $\quad$ Suplemento Nutricional Oral

MMII: membros inferiores

TC: $\quad$ trato gastrointestinal ;

TNE: $\quad$ terapia nutricional enteral ;

TNP: $\quad$ terapia nutricional parenteral

Vo: $\quad$ via oral

ONTOP: $\quad$ Optimal Evidence-Based Non-Drug Therapies in Older People

EMTN: $\quad$ equipe multidisciplinar de terapia nutricional

QVRS: Qualidade de Vida Relacionada à Saúde

CFP: $\quad$ posição cefalo-podálica 


\section{Sumário}

Diga Não à Lesão por Pressão

Say No to Pressure Ulcer

Antonio Carlos L. Campos

Campanha Diga Não à Lesão por Pressão

2

Say No to Pressure Ulcer Campaign

Liane Brescovici Nunes de Matos, Silvia Maria Fraga Piovacari, Ricardo Ferrer, Juliana Tepedino Martins

Alves, Thaisa Assis, Amanda C. M. A. G. Brandão, Melina Gouveia Castro, Guilherme Duprat Ceniccola, Haroldo Falcão Ramos da Cunha, Maria Carolina Conçalves Dias, Rogério Dib, Diana Borges Dock-

Nascimento, Maria Emília Gaspar Ferreira, Ivens Willians Silva Giacomassi, Rodrigo Costa Conçalves, Thiago

José Martins Gonçalves, Claudia Satiko Takemura Matsuba, Julieta Regina Moraes, Mara Rubia de Moura,

Priscila Barsanti de Paula Nogueira, Bruna Fernanda Camargo Silva Parra, Paula Pexe-Machado, Ricardo

Tadeu Prete, Fernanda Antunes Ribeiro, Renata Alves dos Santos, Glaucia Fernanda Corrêa Gaetano Santos, Letícia Faria Serpa, Mariana Fernandes Cremasco de Souza, Diogo Oliveira Toledo, Clarissa Martins Zambelli, Adriano Antonio Mehl, Antonio Carlos Ligocki Campos 


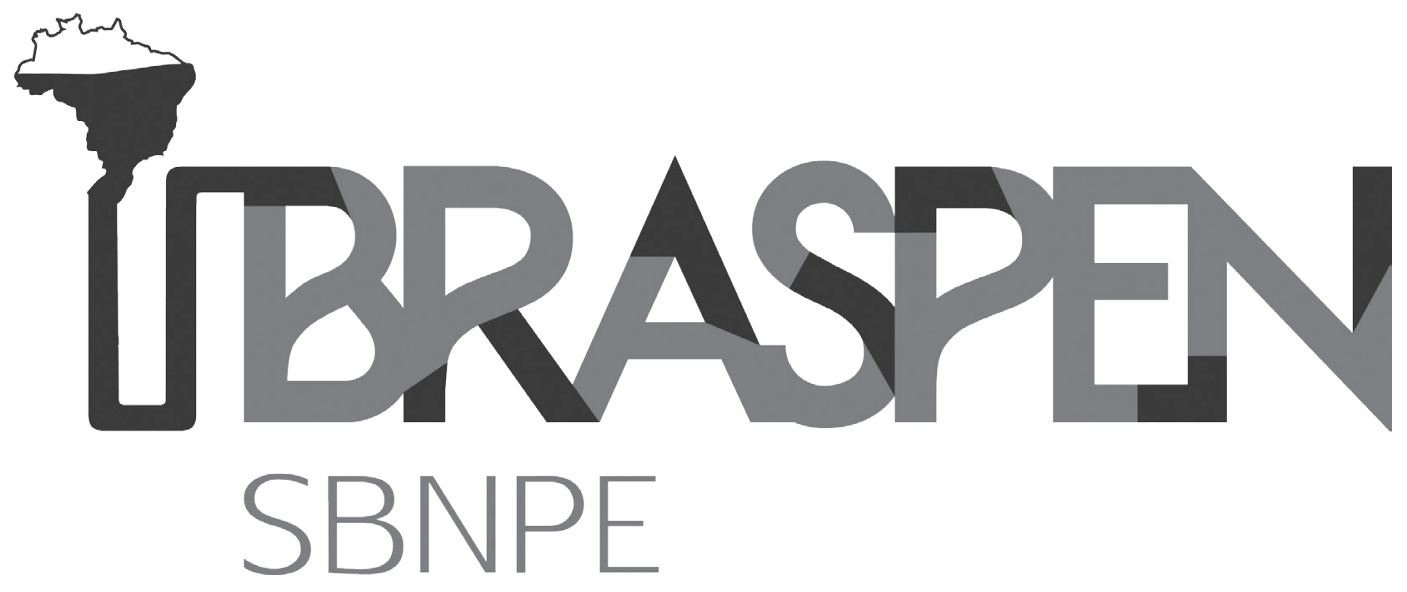

CITE OS ARTIGOS DO BRASPEN JOURNAL NAS SUAS PUBLICAÇÕES. AS CITAÇÕES ROBUSTECERÃO NOSSAS CHANCES DE INDEXAÇÃO. 


\section{Editorial}

\section{DIGA NÃO À LESÃO POR PRESSÃO}

SAY NO TO THE PRESSURE INJURY

O presente suplemento do BRASPEN Journal apresenta a campanha "DIGA NÃO À LESÃO POR PRESSÃO”. Trata-se de mais uma excelente iniciativa da Diretoria da Braspen para estimular a padronização dos cuidados multiprofissionais de pacientes graves. As lesões por pressão são infelizmente bastante frequentes, porém potencialmente preveníveis. Causam grande desconforto e podem ter consequências desastrosas, inclusive com óbito do paciente. Além disso, elevam acentuadamente o tempo de internação e os custos do tratamento. Portanto, podem ser considerados eventos iatrogênicos associados aos cuidados de saúde.

O conceito clássico de Medicina Baseada em Evidências, introduzido por Fields et al há algumas décadas, foi subsequentemente estendido para a área da Nutrição Clínica por Ballesteros y Vidal com a expressão "Nutrição Baseada em Evidências", definida como "conjunto de recomendações desenvolvidas de maneira sistemática para ajudar os profissionais de saúde na tomada de decisões sobre qual a melhor intervenção nutricional para resolver um problema clínico específico". A presente Campanha, a despeito de não se caracterizar como uma Diretriz de Nutrição Clínica, não deixa de ser um referencial extremamente útil para a tomada de decisões e deverá ser amplamente divulgada entre os profissionais de saúde.

Neste aspecto, é com enorme satisfação que a comunidade brasileira dedicada à Nutrição Clínica e Terapia Nutricional recebe mais essa Campanha, desenvolvida com um método mnemônico com a palavra CICATRIZAÇÃO, em que cada letra propõe passos que auxiliam na identificação do risco, avaliação, tratamento e acompanhamento da lesão por pressão, além das medidas para a sua prevenção. Os doze passos abordados, baseados em cada letra da palavra CICATRIZAÇÃO, são apresentados de forma lógica e bastante prática: há um referencial teórico que antecede a abordagem prática de cada tópico. O texto é acompanhado de inúmeros quadros e tabelas que facilitam muito o seu entendimento. Também foram incluídas inúmeras fotografias que exemplificam muito bem a classificação das lesões por pressão, além de situações específicas nas quais são demonstradas situações de risco frequentemente encontradas na prática clínica. Essa campanha tem um valor prático imenso, pois formula linhas de tratamento baseadas no conhecer multidisciplinar, envolvendo médicos, nutricionistas, enfermeiros e farmacêuticos. Além disso, promove a uniformização das práticas de terapia nutricional, racionaliza o uso da terapia nutricional e, consequentemente, reduz custos.

Nas últimas décadas, a terapia nutricional tem demonstrado sua importância, principalmente por contribuir de maneira positiva nos desfechos clínicos de pacientes desnutridos ou em risco de desnutrição. Como a terapia nutricional deve ser parte integral do tratamento de pacientes graves, desnutridos ou em risco de desnutrição, a mesma precisa ser bem implementada para que apresente os benefícios desejados. Nesse sentido, existe a necessidade de haver equipes multiprofissionais dedicadas e que exerçam sua atividade profissional dentro dos mais elevados padrões de qualidade na assistência nutricional.

Parabéns aos autores dessa importante Campanha por todo o esforço. Estou seguro de que servirão para atualizar os conceitos e uniformizar os cuidados nutricionais dos pacientes críticos.

Com certeza, a tenacidade, competência e determinação da atual diretoria da BRASPEN, bem como de futuras diretorias, envidarão todos os esforços para que outras Campanhas como essa sejam criadas.

\section{Prof. Dr. Antonio Carlos L. Campos}

Professor Titular de Cirurgia do Aparelho Digestivo da Universidade Federal do Paraná. Coordenador do Programa de PósGraduação em Clínica Cirúrgica da UFPR. Ex-Presidente da Braspen e da Felanpe 


\title{
Campanha Diga Não à Lesão por Pressão
}

\author{
Say No to Pressure Ulcer Campaign
}

\section{DOI: 10.37111/braspeni.diganaoalesao2020}

\author{
Liane Brescovici Nunes de Matos 1,2,3 \\ Silvia Maria Fraga Piovacari ${ }^{4}$ \\ Ricardo Ferrer ${ }^{2}$ \\ Juliana Tepedino Martins Alves ${ }^{5,6}$ \\ Thaisa Assis ${ }^{7}$ \\ Amanda C. M. A. G. Brandão ${ }^{4}$ \\ Melina Gouveia Castro ${ }^{4}$ \\ Guilherme Duprat Ceniccola ${ }^{8}$ \\ Haroldo Falcão Ramos da Cunha ${ }^{9}$ \\ Maria Carolina Gonçalves Dias ${ }^{10}$
}

\author{
Rogério $\mathrm{Dib}^{4}$ \\ Diana Borges Dock-Nascimento"1 \\ Maria Emília Gaspar Ferreira ${ }^{4}$ \\ Ivens Willians Silva Giacomassi ${ }^{12}$ \\ Rodrigo Costa Gonçalves ${ }^{13,14}$ \\ Thiago José Martins Gonçalves ${ }^{15}$ \\ Claudia Satiko Takemura Matsuba ${ }^{16}$ \\ Julieta Regina Moraes ${ }^{4}$ \\ Mara Rubia de Moura ${ }^{17}$ \\ Priscila Barsanti de Paula Nogueira ${ }^{4}$ \\ Bruna Fernanda Camargo Silva Parra ${ }^{2}$
}

\author{
Paula Pexe-Machado ${ }^{18}$ \\ Ricardo Tadeu Prete ${ }^{16}$ \\ Fernanda Antunes Ribeiro ${ }^{4}$ \\ Renata Alves dos Santos \\ Glaucia Fernanda Corrêa Gaetano Santos ${ }^{4}$ \\ Letícia Faria Serpa $^{19}$ \\ Mariana Fernandes Cremasco de Souza ${ }^{4}$ \\ Diogo Oliveira Toledo ${ }^{2,4}$ \\ Clarissa Martins Zambelli $i^{20,21}$ \\ Adriano Antonio Mehl ${ }^{22}$ \\ Antonio Carlos Ligocki Campos ${ }^{23}$
}

\section{Unitermos: \\ Lesão por pressão. Fatores de risco. \\ Desnutrição. Cicatrização.}

\section{Keywords:}

Pressure ulcer. Risk factors. Malnutrition. Wound healing.

\section{Endereço para correspondência:}

Liane Brescovici Nunes de Matos

Rua Abílio Soares, 233 - cj 144 - Paraíso

- São Paulo, SP, Brasil - CEP: 04005-000

E-mail: lianenunes@yahoo.com.br

\section{Submissão:}

20 de fevereiro de 2020

\section{Aceito para publicação:}

24 de abril de 2020

\begin{abstract}
RESUMO
A lesão por pressão (LP) pode ser classificada de acordo com o comprometimento tecidual, etiologia, tempo de cicatrização e características teciduais. Atualmente, é o terceiro tipo de evento adverso mais notificado pelos núcleos de segurança do paciente dos serviços de saúde do País, de acordo com o Relatório Nacional de Incidentes Relacionados à Assistência à Saúde. A classificação e a identificação dos fatores de risco são fundamentais para implementação de ações preventivas e terapêuticas nos pacientes suscetíveis. $O$ estado nutricional é uma peça fundamental para a prevenção e o desenvolvimento de $L P$, tornando essencial a integração da equipe interdisciplinar na prevenção e no tratamento da LP, com foco na qualidade, segurança e experiência do paciente. Nesse sentido, o objetivo dessa campanha é difundir conhecimento, oportunizando suporte técnico ao cuidado do paciente e reduzir os índices de LP. Foi desenvolvido um mnemônico com a palavra "CICATRIZAÇÃO", em que cada letra propõe passos que auxiliam na identificação do risco, avaliação, tratamento e acompanhamento da LP. Dessa maneira, a sequência dos 12 passos estabelecidos nesta campanha é sugerida como medida para auxiliar aos profissionais da saúde neste manejo.
\end{abstract}

\section{ABSTRACT}

Pressure ulcer (PU) can be classified according to tissue impairment, etiology, healing time and tissue characteristics. Currently, it is the third type of adverse event most reported by the patient safety centers of health services in the country, according to the National Report of Incidents Related to Health Care. The classification and the identification of risk factors are essential for implementation of preventive and therapeutic actions in susceptible patients. Nutritional status is a fundamental piece for the prevention and development of PU, making it essential to integrate the interdisciplinary team in the prevention and treatment of PU, with a focus on quality, safety and patient experience. In this sense, the objective of this campaign is to disseminate knowledge, providing technical support for patient care and reducing PU rates. A mnemonic was developed with the word "CICATRIZATION", in which each letter proposes steps that help in the identification of risk, assessment, treatment and monitoring of PU. Thus, the sequence of 12 steps established in this campaign is suggested as a measure to assist health professionals in this management.

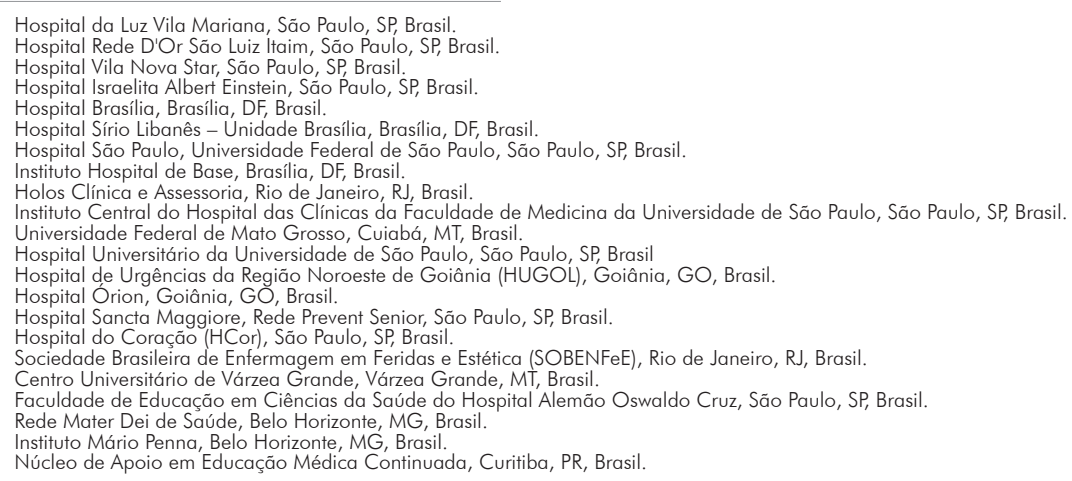




\section{INTRODUÇÃO}

A lesão por pressão (LP) é definida como um dano localizado na pele ou tecidos moles subjacentes, como resultado de pressão, ou pressão em combinação com cisalhamento, geralmente sobre uma proeminência óssea ou relacionado ao uso de dispositivo médico ou a outro artefato'.

A pele é considerada o maior e mais pesado órgão do corpo humano. Está exposta aos fatores ambientais e, por isso, sujeita a constantes agressões, sendo a primeira barreira de proteção do organismo contra agentes externos. Um sistema tegumentar intacto fornece proteção contra patógenos, irritantes, perda de líquidos, bem como regulação da temperatura corporal, o que torna sua integridade e capacidade de reparação essenciais para a sobrevivência do indivíduo².

De acordo com o Relatório Nacional de Incidentes Relacionados à Assistência à Saúde ${ }^{3}$, as notificações de lesões por pressão corresponderam a $17,6 \%$ dos eventos adversos notificados, no período de janeiro de 2014 a julho de 2017, ao Sistema Nacional de Vigilância Sanitária, totalizando 23.722 notificações, uma média aproximada de 18 eventos por dia. Durante este período, a LP foi o terceiro tipo de evento adverso mais frequentemente notificado pelos núcleos de segurança do paciente dos serviços de saúde do País. Ainda, de acordo com o referido relatório, foram notificados 3.771 never events (eventos que, pelo impacto e risco à vida do paciente, nunca deveriam ocorrer em serviços de saúde), sendo que $94,6 \%$ deles foram decorrentes de LP estágio 3 ou estágio 4. Quanto aos óbitos, foram notificados 766 óbitos por eventos adversos no período, sendo que destes, 34 pacientes foram a óbito devido às complicações da $\mathrm{LP}^{3}$.

Consideradas entre os eventos iatrogênicos mais comuns associados aos cuidados de saúde, as lesões por pressão são frequentemente difíceis de curar, dolorosas e impactam negativamente na qualidade de vida do indivíduo, com aumento do risco de infecção, agravamento do quadro clínico, aumento da demanda de cuidados, tempo prolongado de internação e aumento da mortalidade ${ }^{4,5}$. Deste modo, a LP compromete os indicadores de segurança e de qualidade assistencial, além do tratamento e manejo das complicações dela decorrentes serem muito dispendiosos para os serviços de saúde ${ }^{6}$.

Portanto, a campanha visa difundir conhecimento por meio de abordagem ampla e prática, a fim de dar suporte técnico ao cuidado do paciente e, assim, reduzir os índices de LP. Para facilitar a assimilação dos conceitos foi desenvolvido um método mnemônico com a palavra "CICATRIZAÇÃO", em que cada letra propõe passos que auxiliam na identificação do risco, avaliação, tratamento e acompanhamento da LP, conforme apontado no Quadro 1.
Quadro 1 - Método mnemônico dos 12 passos para o combate da lesão por pressão.

C Conhecer o risco de lesão por pressão

I Inspecionar a integridade cutânea

C Classificar o estágio da lesão por pressão

A Avaliar o estado nutricional

T Traçar metas nutricionais e de hidratação

R Reposicionar no leito de 2 em 2 horas

I Implementar protocolos de terapia nutricional

Z Zerar a ocorrência por meio de ações de prevenção

A Avaliar necessidade de nutrientes específicos

Ç Capacitar e conscientizar equipe, familiares e pacientes

Ã Anotar e registrar a evolução da lesão por pressão

0 Orientar a alta hospitalar

Os 12 passos para o combate à LP serão detalhados nos tópicos abaixo:

\section{CONHECER O RISCO DE LESÃO POR PRESSÃO}

\section{Referencial Teórico}

A identificação dos principais fatores de risco é fundamental para implementação de ações preventivas e terapêuticas nos pacientes suscetíveis. A causa do desenvolvimento da LP é multifatorial e inclui fatores intrínsecos - inerentes à fragilidade dos pacientes; e extrínsecos - relacionados ao meio e às exposições sofridas pelo paciente ao longo de sua internação e cuidados hospitalares?'.

A maioria dos métodos de avaliação de risco foi baseada em revisão da literatura, opinião de especialistas e/ou adaptação de uma ferramenta existente. As escalas mais utilizadas são a Escala de Norton, o Escore de Waterlow e a Escala de Braden. Ainda, utiliza-se a Escala de Avaliação do Risco de Desenvolvimento de Lesão por Pressão em Cuidados Intensivos (EVARUCI) para pacientes de unidade de terapia intensiva (UTI) e a Escala de Avaliação de Risco para o Desenvolvimento de Lesões Decorrentes do Posicionamento Cirúrgico (ELPO) para pacientes no centro cirúrgico ${ }^{7,8}$.

As ferramentas de avaliação de risco não incluem necessariamente a avaliação de todos os fatores que podem aumentar o risco de desenvolvimento de LP' . Dentre os fatores mais prevalentes, destacam-se:

- Redução na mobilidade e atividade física - favorecem o aumento de pressão sobre proeminências ósseas, em especial pacientes acamados e com lesão medular ${ }^{1,9}$; 
- Presença de edema e/ou condições que alteram a perfusão e oxigenação dos tecidos, como diabetes mellitus, doença vascular e tabagismo9-11;

- Estado nutricional de desnutrição, sarcopenia ou obesidade $^{1,12}$;

- Aumento na umidade da pele, comumente observados nos pacientes com incontinência urinária e fecal ${ }^{11}$;

- Elevada temperatura corporal ${ }^{12}$;

- Déficit na percepção sensorial da pele ${ }^{12}$;

- Alterações laboratoriais, como queda da hemoglobina, com consequente redução da oxigenação dos tecidos, aumento do marcador inflamatório proteína $C$ reativa (PCR), leucopenia e hipoalbuminemia, ocasionando edema e alterações na perfusão tecidual ${ }^{13}$;

- Presença de doenças prévias, feridas crônicas, uso de medicações, infecção e alteração do nível de consciência ${ }^{14-17}$;

- Tempo cirúrgico elevado e imobilidade no perioperatório';

- Gravidade da doença - pacientes críticos, agravados pelo tempo de internação na UTI, ventilação mecânica, uso de vasopressores $8,14,15,18,19$;

- Pacientes em cuidados paliativos';

- Pacientes em transportes prolongado, por exemplo, em ambulâncias';

- Neonatos e crianças, devido à imaturidade da pele, com repercussão na perfusão tecidual' ;

- Uso de dispositivos médicos, mais comumente dispositivos para suporte ventilatório'.

Consideram-se de alto risco para LP os pacientes que apresentem múltiplos fatores que afetem: condições mecânicas, como pontos de aumento da pressão na pele, fricção ou cisalhamento e fatores relacionados às características morfológica e fisiológica dos tecidos, como no paciente idoso $1,10,12,19,20$.

\section{Abordagem Prática}

- Realizar avaliação multiprofissional do paciente internado, elencando os fatores de risco para desenvolvimento de $L P$;

- Reavaliar a presença de fatores de risco, alteração do quadro clínico e gravidade da doença de base diariamente;

- Sugere-se a utilização da Escala de Braden ${ }^{8}$ em todos os pacientes intra-hospitalares ou em internação domiciliar.

O Quadro 2 apresenta a categorização dos fatores de risco que favorecem o surgimento da LP.
Quadro 2- Fatores de risco para o desenvolvimento de lesão por pressão.

\begin{tabular}{|c|c|c|}
\hline Fator de risco & $\begin{array}{c}\text { Exposição } \\
\text { ao dano } \\
\text { mecânico }\end{array}$ & $\begin{array}{l}\text { Suscetibilidade } \\
\text { e tolerância } \\
\text { individual }\end{array}$ \\
\hline $\begin{array}{l}\text { Atividade e limitação de } \\
\text { mobilidade }\end{array}$ & $\mathrm{X}$ & \\
\hline Condição atual da pele & & $X$ \\
\hline $\begin{array}{l}\text { Perfusão, circulação e } \\
\text { oxigenação tecidual }\end{array}$ & & $X$ \\
\hline Indicadores nutricionais & & $X$ \\
\hline Umidade da pele & $X$ & $X$ \\
\hline Temperatura corporal & & $X$ \\
\hline Idade avançada & $\mathrm{X}$ & $X$ \\
\hline Limitação de percepção sensorial & $x$ & \\
\hline Marcadores sanguíneos & & $X$ \\
\hline Condição geral e saúde mental & $\mathrm{X}$ & $X$ \\
\hline \multicolumn{3}{|c|}{ Fatores de risco para populações específicas } \\
\hline Pacientes cirúrgicos & $\mathrm{X}$ & $X$ \\
\hline Pacientes críticos & $X$ & $X$ \\
\hline Neonatos e crianças & $X$ & $X$ \\
\hline
\end{tabular}

Fonte: European Pressure Ulcer Advisory Panel: International Guideline, 2019².

\section{INSPECIONAR A INTEGRIDADE CUTÂNEA}

\section{Referencial Teórico}

A inspeção inicial da integridade cutânea é ação principal e essencial na prevenção, classificação, diagnóstico e tratamento da LP. A condição da pele pode servir como um indicador de sinais precoces de danos, oferecendo oportunidades para identificar e intervir precocemente quando alterações cutâneas são identificadas ${ }^{21}$.

Achados como ressecamento, excesso de umidade, diminuição do estrato córneo ou inflamação enfraquecem a função de barreira da pele e aumentam a suscetibilidade para LP. No ambiente hospitalar, cuidado extra deve ser dado à pele com pigmentação escura, pois LP em estágio 1 são subdetectadas em indivíduos desse grupo, uma vez que alterações de cor são mais difíceis de serem visualizadas. Neste caso, considere a avaliação da temperatura da pele e da umidade subepidérmica como estratégias importantes de avaliação adjunta'.

Neste contexto, o cuidado com a pele íntegra também merece importância. A hidratação da pele e o consequente equilíbrio hídrico cutâneo são fatores fundamentais para garantir a integridade e a função do tecido'. Dentre as principais funções da hidratação, pode-se destacar a reparação da barreira da pele, manutenção da aparência, manutenção da capacidade da barreira lipídica para atrair, manter e redistribuir a água. Existem 
disponíveis diversos insumos que podem ser utilizados no combate à desidratação da pele, como, por exemplo, à base de alfa-hidroxiácidos, glicerina, ureia, propilenoglicol e lipídeos.

\section{Abordagem Prática}

- Garantir que uma avaliação completa da pele seja parte integrante da política de rastreio dos riscos para LP, devendo ser implementada em todas as instituições de saúde? ;

- Realizar avaliação completa da pele em pacientes com risco de desenvolver LP dentro de um prazo máximo de oito horas após a admissão e como parte integrante de todas as avaliações do risco ${ }^{1,22}$;

- Reavaliar de modo contínuo, com base no contexto clínico e no grau de risco do indivíduo: pacientes com moderado a alto risco para LP devem ser reavaliados a cada 24 horas; pacientes de baixo risco para LP podem ser reavaliados a cada 72 horas ou reavaliados imediatamente, caso haja alteração da condição clínica²;

- Avaliar pacientes em internação domiciliar a cada visita da enfermagem assistente ${ }^{22}$;
- Aumentar a frequência das avaliações da pele em resposta a qualquer deterioração do estado geral ${ }^{4}$;

- Inspecionar a pele sob e ao redor dos dispositivos médicos, pelo menos duas vezes ao dia, a fim de identificar sinais de LP no tecido circundante';

- Realizar avaliações da pele mais frequentes (mais de duas vezes ao dia) nos dispositivos de interface direta com a pele, principalmente naqueles indivíduos suscetíveis a alterações de fluidos e/ou com sinais de edema localizado/ generalizado';

- Realizar avaliação antes de o paciente receber alta ;

- Manter os cuidados com a pele íntegra: mantê-la limpa e adequadamente hidratada; proteger da umidade com um produto barreira; aplicar regularmente um hidratante, a fim de promover a hidratação da pele e prevenir condições adversas; não massagear ou esfregar vigorosamente a pele; usar preferencialmente produto fluído, evitando fricção excessiva ${ }^{22}$.

A fim de auxiliar nos cuidados e avaliação da pele, considerações sobre principais pontos a serem avaliados estão descritos no Quadro 3.

Quadro 3 - Abordagem prática na avaliação da integridade da pele.

\begin{tabular}{|c|c|c|}
\hline Avalie & Considere & Definição/padrão de referência \\
\hline Temperatura & $\begin{array}{l}\text { - Há áreas de calor localizado na pele à } \\
\text { - Halpação? } \\
\text { de baixa vascularização }\end{array}$ & $\begin{array}{l}\text { Profissionais treinados conseguem identificar, à palpação, até } 1-3^{\circ} \mathrm{C} \text { de diferença } \\
\text { na temperatura. Estudos mais recentes demonstram que o uso de termômetros } \\
\text { infravermelhos pode ser adjuvante da avaliação manual. }\end{array}$ \\
\hline Consistência & $\begin{array}{l}\text { - A pele está muito fina? } \\
\text { - Há edema perceptível? } \\
\text { - A pele está macerada? }\end{array}$ & $\begin{array}{l}\text { A tolerância tecidual está relacionada com a capacidade tissular de suportar a } \\
\text { pressão, resistindo à deformação e à formação da LP. Ela pode ser alterada por } \\
\text { fatores extrínsecos, como umidade, fricção, cisalhamento; outros, como baixa } \\
\text { pressão arteriolar, além dos fatores de risco. A presença de edema ou mace- } \\
\text { rações exige cuidados adicionais quanto ao risco para desenvolvimento de LP. }\end{array}$ \\
\hline Umidade & $\begin{array}{l}\text { - A umidade da pele é excessiva? } \\
\text { - Há contato com sujidades, exsudações, } \\
\text { - Aecreções, urina ou fezes? } \\
\text { - A pele está ficando macerada? }\end{array}$ & $\begin{array}{l}\text { A umidade aumenta os efeitos da pressão sob a pele, predispondo à necrose e } \\
\text { à maceração, tornando-a mais vulnerável às lesões, além de provocar a adesão } \\
\text { das roupas da cama e do vestuário à pele, potencializando a ação de cisalha- } \\
\text { mento e fricção. } \\
\text { A presença de urina ou fezes muda o pH da pele, além de deixá-la exposta a } \\
\text { substâncias, como a amônia, o que contribui para a quebra de barreira cutânea, } \\
\text { favorecendo a colonização pela microbiota (bactérias principalmente) e predis- } \\
\text { pondo a infecções. }\end{array}$ \\
\hline
\end{tabular}




\section{CLASSIFICAR O ESTÁGIO DA LESÃO POR PRESSÃO}

\section{Referencial Teórico}

A classificação da LP é fundamental para o estabelecimento da abordagem terapêutica. A LP pode ser classificada de acordo com a sua etiologia, comprometimento tecidual, tempo de cicatrização, nível de carga microbiológica, características teciduais, entre outros fatores ${ }^{1,24}$.

Existem diversos instrumentos para avaliação e categorização das lesões de pressão. Utilizaremos a terminologia do Sistema Internacional de Classificação de Lesões por Pressão
(EPUAP/NPIAP/PPPIA), o qual é utilizado para descrever a categoria/estágio da LP? .

Desde 2016 foi proposta a substituição do termo úlcera para lesão, por se entender que lesão descreve melhor esse tipo de dano, pois é referenciado tanto para a pele ainda intacta como na pele já ulcerada ${ }^{25}$.

\section{Abordagem Prática}

Considerando-se a extrema relevância da classificação da LP para a prática clínica, sugere-se a avaliação proposta no Quadro 4.

Quadro 4 - Classificação das lesões por pressão.

\section{Classificação da Lesão}

Estágio 1

\section{Definição}

Pele intacta, área localizada de eritema não-branqueável, que pode aparecer de maneira diferente na pele pigmentada escura. A presença de eritema palpável ou alterações na sensação, temperatura ou turgor podem preceder às alterações visuais. As alterações de cor não incluem descoloração roxa ou marrom, estas podem indicar lesão tissular profunda ${ }^{26}$.

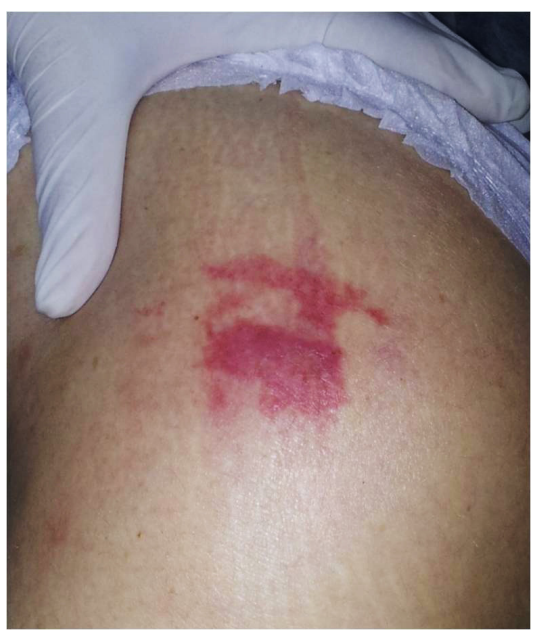

Estágio 2

Perda de espessura parcial da pele com derme exposta. O leito da ferida é viável, rosa ou vermelho, úmido e também pode se apresentar como uma bolha preenchida com exsudato seroso, intacta ou rompida. O tecido adiposo não é visível, assim como o tecido mais profundo. Não há tecido de granulação, descolamento ou escara ${ }^{26}$.
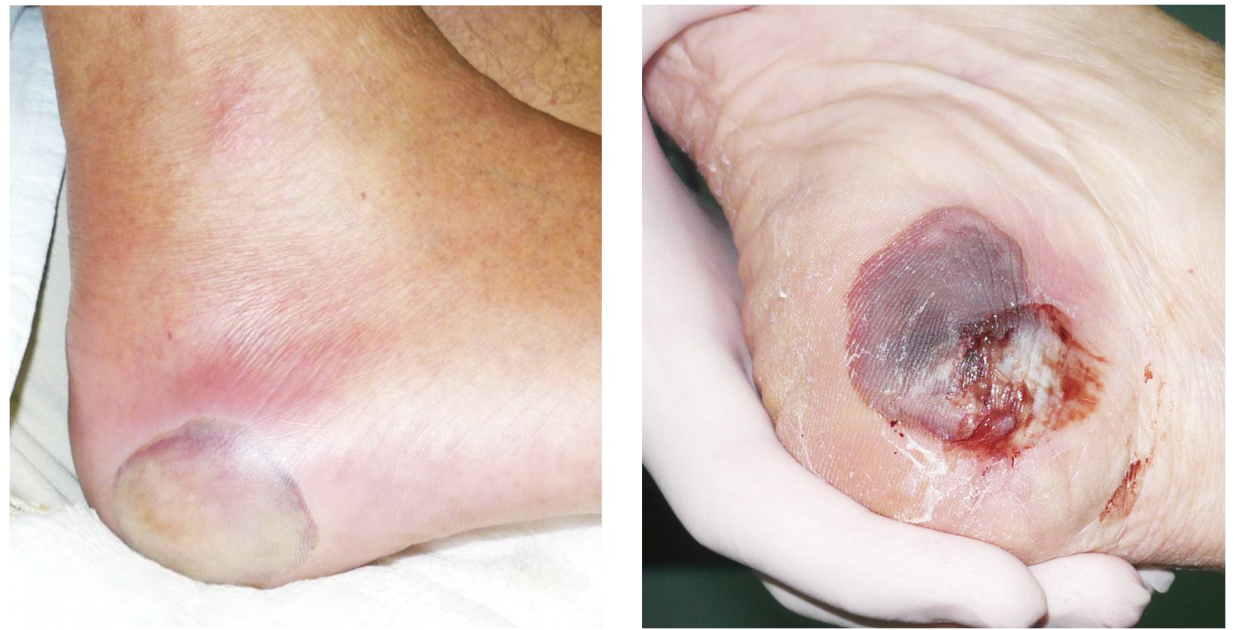
Continuação Quadro 4 - Classificação das lesões por pressão.

\section{Classificação da Lesão}

Estágio 3

\section{Definição}

Há perda de pele em toda sua espessura, na qual o tecido adiposo é visível na lesão e no tecido de granulação, assim como existem bordas enroladas da ferida (epíbole). Descolamento ou escaras podem estar visíveis e áreas de adiposidade significativa podem desenvolver feridas profundas. Tunelização pode ocorrer. Fáscia, músculo, tendão, ligamento, cartilagem ou osso não estão expostos. Se o descolamento ou escaras dificultarem a mensuração da extensão da perda de tecido, esta é uma LP não classificável26.

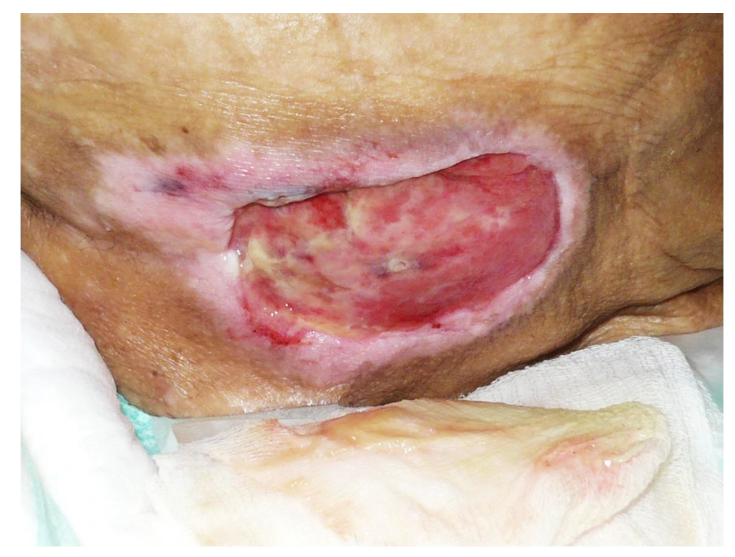

Estágio 4

Não-classificável
Perda total de tecido e pele; com fáscia, músculo, tendão, ligamento, cartilagem e osso que podem estar expostos ou diretamente palpáveis na úlcera. Descolamento ou escaras podem estar visíveis. Frequentemente ocorrem tunelização e enrolamento das bordas (epíbole). A profundidade varia de acordo com a localização anatômica. Se o descolamento ou escaras dificultarem a mensuração da extensão da perda de tecido, esta é uma LP não classificáve $\left.\right|^{26}$.

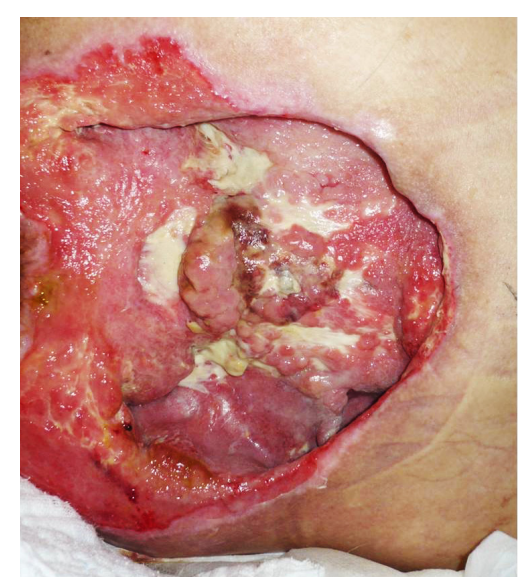

Perda da pele em sua espessura total e perda tissular na qual a extensão do dano não pode ser confirmada porque está encoberta pelo esfacelo ou escara. Ao ser removido (esfacelo ou escara), a lesão por pressão em Estágio 3 ou Estágio 4 ficará aparente. Escara estável (isto é, seca, aderente, sem eritema ou flutuação) em membro isquêmico ou no calcâneo não deve ser removida ${ }^{25}$.

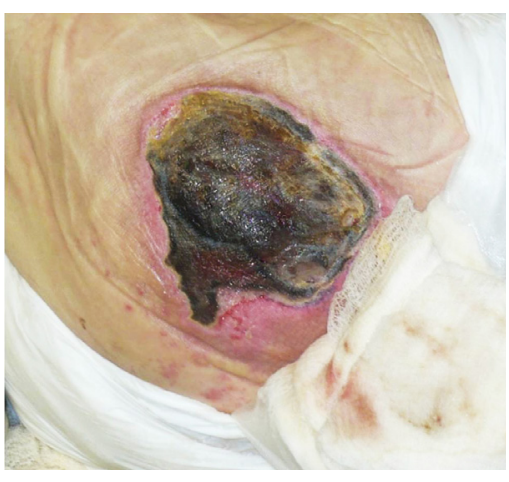


Continuação Quadro 4 - Classificação das lesões por pressão.

\section{Classificação da Lesão}

Lesão tissular profunda

\section{Definição}

Pele intacta ou não, com área localizada e persistente, de descoloração vermelha escura, marrom ou púrpura que não embranquece, ou separação epidérmica que mostra lesão com leito escurecido ou bolha com exsudato sanguinolento. Dor e mudança na temperatura frequentemente precedem às alterações de coloração da pele. A descoloração pode apresentar-se diferente em pessoas com pele de tonalidade mais escura. Essa lesão resulta de pressão intensa e/ou prolongada e de cisalhamento na interface osso-músculo. A ferida pode evoluir rapidamente e revelar a extensão atual da lesão tissular ou resolver sem perda tissular. Quando tecido necrótico, tecido subcutâneo, tecido de granulação, fáscia, músculo ou outras estruturas subjacentes estão visíveis, isso indica lesão por pressão com perda total de tecido (Lesão por Pressão Não Classificável ou Estágio 3 ou Estágio 4). Não se deve utilizar a categoria lesão por pressão tissular profunda (LPTP) para descrever condições vasculares, traumáticas, neuropáticas ou dermatológicas ${ }^{25}$.

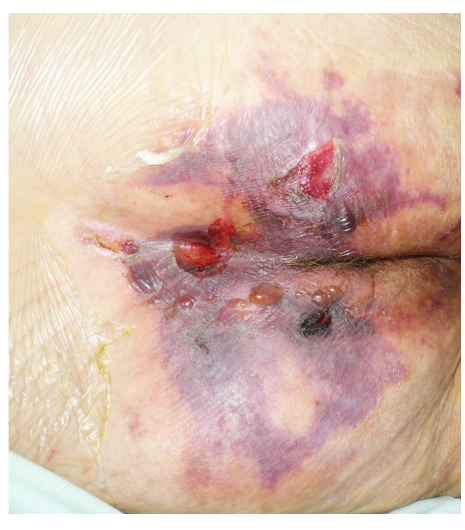

Lesão por pressão membrana mucosa de São lesões por pressão das membranas úmidas que revestem os tratos respiratório, gastrointestinal e geniturinário; causadas principalmente por dispositivos médicos que exercem forças de compressão e cisalhamento sustentadas, tais como: tubos de oxigênio, tubos endotraqueais, cânulas orais, tubos orogástricos e nasogástricos, cateteres urinários, gastrostomias, etc ${ }^{1}$. A lesão por pressão de membrana mucosa não é estagiável.

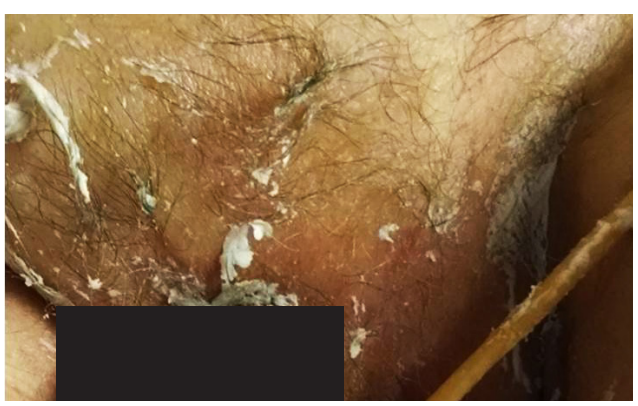

Lesão por pressão relacionada a A LP relacionada a dispositivo médico resulta do uso de dispositivos, equipamentos, mobiliário e objetos do dispositivo médico dia-a-dia que têm causado pressão sobre a pele, como uma consequência não intencional do seu uso terapêutico ou do contato inadvertido com a pele. A LP resultante geralmente apresenta o padrão ou forma do dispositivo. Essa terminologia descreve a etiologia da lesão e não a sua gravidade ou extensão de perda de tecido'.

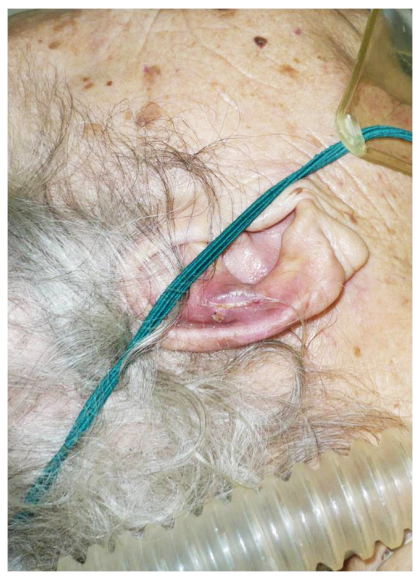




\section{AVALIAR O ESTADO NUTRICIONAL}

\section{Referencial Teórico}

O estado nutricional é determinante para a prevenção e o desenvolvimento de LP, uma vez que todos os tecidos necessitam de macro e micronutrientes para promover crescimento, manutenção e cicatrização. Pacientes desnutridos ou em risco nutricional têm maiores chances de desenvolvimento de LP'. A desnutrição está associada ao desenvolvimento, gravidade e retardo de cicatrização das lesões por pressão ${ }^{27}$. Estudo multicêntrico com 1.412 pacientes idosos demonstrou uma associação significativa entre a presença de lesões por pressão e o risco de desnutrição; utilizando-se da ferramenta Malnutrition Universal Screening Tool (MUST), 36\% dos pacientes com LP estavam em risco de desnutrição ${ }^{28}$. Já um estudo inglês encontrou taxas ainda mais elevadas, $76 \%$ dos idosos hospitalizados com risco para LP eram desnutridos ${ }^{29}$.

Recomenda-se que o risco nutricional seja triado na admissão hospitalar e que a avaliação nutricional seja repetida a cada 7 a 10 dias. É por meio desta que são determinadas as necessidades de proteínas, calorias e dos demais nutrientes que cada paciente deve receber. A hidratação e a nutrição, com oferta calórica e de micronutrientes adequada, contribuem para integridade dos tecidos, inclusive da pele?

Deve-se realizar a triagem com uma ferramenta simples, válida e confiável em 24 a 48 horas da admissão ${ }^{30-33}$. Dentre as ferramentas existentes, a Mini Nutrition Assessment (MAN) considera a própria LP e alguns dos seus fatores de risco na avaliação do risco nutriciona ${ }^{34,35}$. As demais ferramentas, apesar de não incluírem essas informações, também podem ser utilizadas de acordo com a rotina dos serviços ${ }^{1}$. A Escala de Braden, utilizada para avaliar o risco de LP, considera também fatores de risco nutricionais em sua pontuação ${ }^{8}$.

Recomenda-se a avaliação do risco nutricional para todos os pacientes com fatores de risco para LP'. O rastreamento nutricional precoce está associado a uma redução de até $50 \%$ das taxas de LP, além da aceleração da cicatrização da LP, redução da permanência hospitalar e podendo resultar em redução dos custos ${ }^{36}$.

A avaliação nutricional deve incluir história alimentar, medidas antropométricas básicas (peso, altura, índice de massa corpórea), histórico de perda de peso, avaliação de perda de massa muscular, edema, provas de força muscular como dinamometria (handgrip), sinais de deficiência de micronutrientes e habilidade de comer independente $^{37}$. Um estudo com 462 pacientes idosos com fratura de quadril demonstrou que a avaliação de handgrip foi capaz de predizer o risco de desenvolvimento de LP durante a internação hospitalar e em 30 dias após a alta ${ }^{38}$.

\section{Abordagem Prática}

- Realizar triagem nutricional para todos os pacientes internados. Para idosos, sugere-se a ferramenta MAN e, para adultos, a Nutritional Risk Screening 2002 (NRS 2002) ou MUST;

- Incluir na avaliação nutricional: histórico alimentar; medidas antropométricas básicas (peso, altura, índice de massa corpórea); histórico de perda de peso; avaliação de perda de massa muscular; avaliação de edema periférico; avaliação de força com o uso da dinamometria, se possível; avaliação de deficiências de micronutrientes dependendo da história clínica e exame físico e avaliação da habilidade de comer independente.

\section{TRAÇAR METAS NUTRICIONAIS E DE HIDRATAÇÃO}

\section{Referencial Teórico}

A terapia nutricional em pacientes com LP objetiva a regeneração do tecido, favorecendo o processo de cicatrização. A inadequação nutricional é fator de risco para a LP, podendo impactar no desenvolvimento, na gravidade e no prolongamento do processo cicatricial. Uma intervenção que contemple um plano nutricional individualizado é necessária para garantir a adequação de nutrientes e hidratação $0^{1,39,40}$.

As calorias ingeridas pelo paciente, principalmente as provenientes de carboidratos, fornecem energia para o processo de cicatrização da lesão. O fornecimento adequado de calorias é imprescindível na atividade fagocítica, na proliferação celular e na função fibroblástica ${ }^{4}$. A ingestão de calorias deve ser ajustada com base na mudança de peso, grau de obesidade ou conforme o diagnóstico e a condição clínica do paciente. A calorimetria indireta $(\mathrm{Cl})$ é a recomendação padrão ouro para traçar a meta de necessidade energética dos pacientes, entretanto, pelas dificuldades de implementá-la na prática clínica, podem ser utilizadas as fórmulas de bolso validadas'.

Quanto às proteínas, estas participam na neovascularização, proliferação fibroblástica, síntese de colágeno, produção e migração de leucócitos em pacientes com $\mathrm{LP}^{41-43}$. Guidelines internacionais demonstram que constitui uma boa prática clínica o incremento da oferta proteica em indivíduos desnutridos com risco ou presença de LP ${ }^{44-46}$.

A oferta hídrica e a manutenção, em níveis adequados, das proteínas séricas são necessárias para promover 
cicatrização satisfatória, situação nem sempre encontrada em idosos frágeis e em cuidados de fim de vida ${ }^{47,48}$. Estes fatores têm papel importante no balanço hidroeletrolítico, turgor da pele, perfusão tecidual e temperatura corporal, além de servirem como solvente para vitaminas, minerais, glicose e outros nutrientes. A desidratação prejudica as funções vitais de circulação, diminuindo a oxigenação dos tecidos. A menor perfusão e oxigenação tecidual reduz a taxa de metabolismo e energia do tecido, predispondo à hipoxemia e à disfunção orgânica, que contribuem para a formação da LP ${ }^{48,49}$. Condições de hipertermia, êmese, diarreia, sudorese elevada ou feridas com alta exsudação e pacientes com alto consumo proteico requerem ofertas hídricas adicionais, que devem ser avaliadas individualmente?

\section{Abordagem Prática}

O Quadro 5 sugere regras de bolso para cálculo das necessidades energéticas e proteicas baseadas nas últimas diretrizes nacionais e internacionais, bem como as vias de administração da terapia nutricional.

\section{REPOSICIONAR NO LEITO DE 2 EM 2 HORAS}

\section{Referencial Teórico}

A necessidade de promover a mobilização do paciente para prevenção e auxílio no tratamento de LP está muito bem estabelecida na prática clínica. Dados da literatura têm demonstrado a importância da implantação de protocolos, tais como: reposicionamento no leito personalizado e sistemático a cada 2 horas, manobras de alívio da pressão quando o paciente estiver sentado, hidratação e higiene adequadas da pele, proteção de pontos suscetíveis que normalmente são aqueles com maior saliência óssea ou locais onde já ocorreram lesões no passado'. Estes pontos podem estar localizadas nos membros inferiores (trocânter, maléolo e calcâneo), na pelve e, apesar de menos frequentes, nos membros superiores (cotovelos, ombros e escápulas), de modo que todos exigem monitoramento contínuo.

É conhecido que a mobilização e o reposicionamento procuram redistribuir a pressão sobre a pele, principalmente sobre as proeminências ósseas, mantendo adequada circulação sanguínea e reduzindo a magnitude da força, além de ser considerada uma manobra para a vitalidade tecidual ${ }^{53}$.

Quadro 5 - Necessidades nutricionais e vias de administração da terapia nutricional na lesão por pressão.

\begin{tabular}{|c|c|c|}
\hline & Risco de lesão por pressão & Lesão por pressão instalada \\
\hline Calorias no Paciente Crítico* & $\begin{array}{l}15 \text { a } 20 \mathrm{kcal} / \mathrm{kg} / \mathrm{dia} \text { do } 1^{\circ} \text { ao } 3^{\circ} \mathrm{dia} ; \\
25 \text { a } 30 \mathrm{kcal} / \mathrm{kg} / \mathrm{dia} \text { após o } 4^{\circ} \text { dia dos pacientes em } \\
\text { recuperação }{ }^{51}\end{array}$ & $\begin{array}{l}15 \text { a } 20 \mathrm{kcal} / \mathrm{kg} / \mathrm{dia} \text { do } 1^{\circ} \text { ao } 3^{\circ} \mathrm{dia} ; \\
25 \text { a } 30 \mathrm{kcal} / \mathrm{kg} / \mathrm{dia} \text { após o } 4^{\circ} \text { dia dos pacientes em } \\
\text { recuperação }{ }^{51}\end{array}$ \\
\hline Calorias no Paciente Obeso* & $\begin{array}{l}11-14 \mathrm{kcal} / \mathrm{kg} / \text { dia do peso real para pacientes com } \\
\text { IMC entre } 30-50 \mathrm{~kg} / \mathrm{m}^{2} \\
22-25 \mathrm{kcal} / \mathrm{kg} / \text { dia do peso ideal, para paciente com } \\
\mathrm{IMC}>50 \mathrm{~kg} / \mathrm{m}^{2} 51\end{array}$ & $\begin{array}{l}11-14 \mathrm{kcal} / \mathrm{kg} / \text { dia do peso real para pacientes com IMC } \\
\text { entre } 30-50 \mathrm{~kg} / \mathrm{m}^{2} \\
22-25 \mathrm{kcal} / \mathrm{kg} / \text { dia do peso ideal, para paciente com IMC } \\
>50 \mathrm{~kg} / \mathrm{m}^{2}{ }^{51}\end{array}$ \\
\hline Proteínas & $\begin{array}{l}1,25-1,5 \mathrm{~g} \text { de proteína } / \mathrm{kg} / \mathrm{dia}^{1} \\
\text { Em pacientes renais, avaliar a condição clínica }\end{array}$ & $\begin{array}{l}1,5-2 \mathrm{~g} \text { de proteína } / \mathrm{kg} / \mathrm{dia}^{1,51} \\
\text { Em pacientes renais, avaliar a condição clínica }\end{array}$ \\
\hline $\begin{array}{l}\text { Suplemento Nutricional } \\
\text { Oral (SNO) }\end{array}$ & $\begin{array}{l}\text { Se baixa aceitação alimentar (inferior a } 60 \% \text { das } \\
\text { necessidades nutricionais), avaliar a necessidade } \\
\text { de introdução de SNO hiperproteico no contexto } \\
\text { da dieta ofertada }{ }^{1,52}\end{array}$ & $\begin{array}{l}\text { Introduzir suplemento nutricional oral específico para } \\
\text { cicatrização (contendo nutrientes específicos: zinco, } \\
\text { arginina, carotenoides, vitaminas } A, C \text { e E })^{1}\end{array}$ \\
\hline Nutrição Enteral & $\begin{array}{l}\text { Se aceitação alimentar com SNO for menor que } \\
60 \% \text { das necessidades nutricionais, a terapia } \\
\text { nutricional enteral está indicada }{ }^{1,52}\end{array}$ & $\begin{array}{l}\text { Se aceitação alimentar com SNO for menor que } 60 \% \\
\text { das necessidades nutricionais, a terapia nutricional } \\
\text { enteral está indicada }{ }^{1,50,51}\end{array}$ \\
\hline Nutrição Parenteral & $\begin{array}{l}\text { Se impossibilidade de utilização do trato } \\
\text { gastrointestinal, a terapia nutricional parenteral } \\
\text { está indicada }{ }^{1,50}\end{array}$ & $\begin{array}{l}\text { Se impossibilidade de utilização do trato } \\
\text { gastrointestinal, a terapia nutricional parenteral } \\
\text { está indicada1,50 }\end{array}$ \\
\hline
\end{tabular}




\section{Abordagem Prática}

As principais recomendações quanto à mudança de decúbito são $0^{1,54-56}$ :

- Avaliar inicialmente a pele, a fim de direcionar a frequência do reposicionamento no leito, considerando: nível de atividade, mobilidade, condição clínica, condição da pele, conforto e dor;

- Inspecionar regularmente a pele e proeminências ósseas durante o reposicionamento, com registro em prontuário ao menos uma vez ao dia;

- Incentivar o autocuidado para aqueles que conseguem se reposicionar. Envolver cuidadores e familiares neste processo é bastante aplicável na prática clínica e aparenta ter melhor aderência ao cuidado;

- Registrar a frequência de mudança de decúbito, assim como a posição e a angulação;

- Priorizar decúbito a $30^{\circ}$ na posição semi-fowler e rotação máxima de $30^{\circ}$ para as laterais (alternando lado direito, esquerdo e dorsal);

- Restringir o tempo em posição sentado sem alívio de pressão, pois o peso do corpo aumenta a pressão das tuberosidades isquiáticas. Na prática clínica, são adotadas ações para amenizar os pontos de pressão quando em poltrona/cadeira, como permanecer por 1 hora com os membros inferiores (MMII) elevados e 1 hora com os MMII apoiados no chão, não ultrapassando o tempo de máximo de 2 horas;

- Após o posicionamento, verificar os pontos de pressão sobre dispositivos, como sondas e drenos, recomenda-se o uso de curativos preventivos para alívio de pressão em casos de difícil controle;

- Avaliar regularmente desconforto e dor. Na vigência destes, reconsidere a frequência e o método de reposicionamento;

- Avaliar a necessidade de analgesia pré-mobilização por ao menos 20 a 30 minutos, em casos de dor intensa;

- Pacientes com instabilidade hemodinâmica podem e devem ser mobilizados, desde que recupere seus parâmetros em torno de 10 a 20 minutos após o reposicionamento no leito (ajuste da pressão ortostática e linha de base). Em casos selecionados, mobilizar o paciente gradualmente a $15^{\circ}$, no primeiro minuto, e se tolerância, atingir $30^{\circ}$;

- $O$ uso de travesseiros e coxins para apoiar os braços e pernas tem bons resultados em pacientes gravemente enfermos por minimização de cisalhamento;

- Utilizar elevadores mecânicos e lençóis de transferência, com o intuito de reduzir o risco de cisalhamento;

Quando em prona, proceder à aplicação de curativos preventivos em áreas de maiores pontos de pressão, como proeminências ósseas, avaliando frequentemente a região facial;
- Priorizar o conforto em pacientes em cuidados de fim de vida;

- Não são recomendados dispositivos auxiliares em forma de anel ou argola (almofadas ou assentos circulares) por criarem áreas de elevada pressão com risco de dano aos tecidos, tampouco almofadas em pele de carneiro sintética, sacos de fluidos intravenosos ou luvas cheias de água para elevar os calcâneos.

\section{IMPLEMENTAR PROTOCOLOS DE TERAPIA NUTRICIONAL}

\section{Referencial Teórico}

Os protocolos clínicos constituem os melhores métodos para integrar e sistematizar as boas práticas de cuidados ao doente. Para construção de protocolos devemos levar em consideração a população alvo, bem como o perfil da instituição envolvida, visando à criação de modelo padronizado que auxilie e uniformize as condutas dentro da instituição. Algoritmos, prescrições médicas, dietéticas e de enfermagem podem ser incorporados aos protocolos e permitir a expectativa de resultados bem definidos. De maneira genérica, protocolos clínicos para uso local devem considerar a literatura médica relevante e as diretrizes e padrões de cuidados estabelecidos por sociedades profissionais nacionais ou internacionais.

No contexto da terapia nutricional, a implantação de protocolos parece melhorar de maneira expressiva a qualidade da terapia nutricional realizada, bem como uniformizar as condutas da equipe assistente no que se refere à terapia nutricional adotada. Para que haja aderência aos protocolos é preciso que sua implantação contemple a interação de vários fatores associados ao tipo de protocolo, processo de implantação, características da instituição e perfil da equipe. $\bigcirc$ sucesso da implantação e execução de um protocolo de conduta nutricional reside na sua simplicidade e personalização. Para maior efetividade, devem contemplar o embasamento científico das recomendações oferecidas por diretrizes, adaptação à realidade de cada serviço, levando em consideração clareza, concisão, formato e o fácil manuseio ${ }^{57}$.

A implantação de protocolos de conduta em terapia nutricional pode ser uma alternativa relevante para melhorar resultados. No escopo da terapia nutricional, os conceitos de gestão e garantia de qualidade podem ser traduzidos pela necessidade de protocolos, de manuais de procedimentos, de indicadores e de verificações no cumprimento das rotinas ${ }^{58}$. Segundo estudo que investigou o uso de um protocolo nutricional para pacientes com LP Estágio 2 ou 3 , a avaliação nutricional se associou à melhora da cicatrização da $L P^{1}$. 


\section{Abordagem Prática}

- Apresenta-se sugestão de protocolos em formato de fluxograma (Figura 1), estes foram adaptados das referências: EMTN em Prática HIAE ${ }^{50}$, EPUAP/NPIAP/PAN PACIFIC $2019^{1}$ e Diretriz BRASPEN de Envelhecimento $2019^{59}$.

\section{ZERAR A OCORRÊNCIA POR MEIO DE AÇÕES DE PREVENÇÃO}

\section{Referencial Teórico}

Uma abordagem em nível gerencial, capaz de enxergar o processo assistencial no nível de toda organização hospitalar,

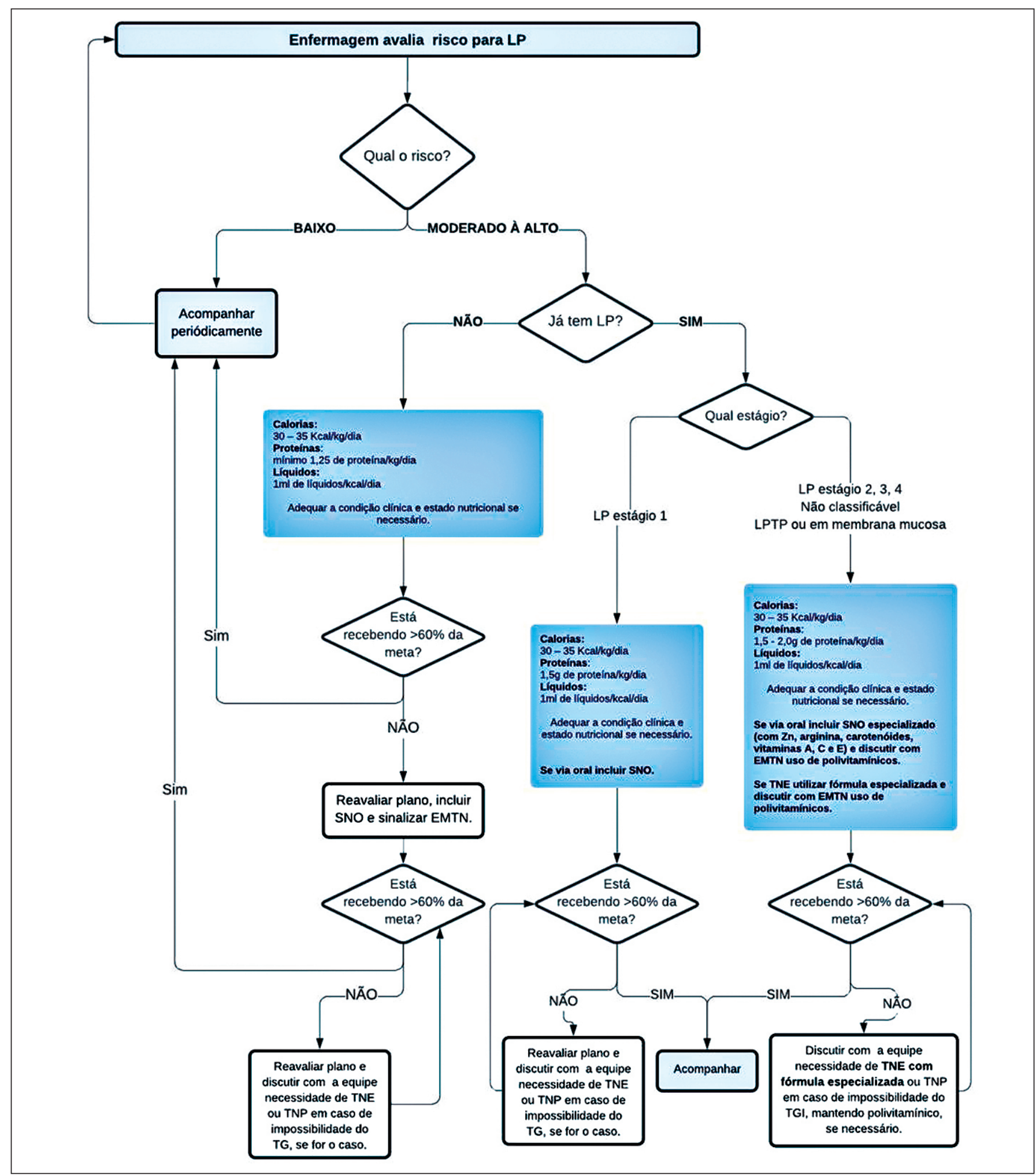

Figura 1 - Protocolo de avaliação e tratamento da lesão por pressão para pacientes estáveis. 
é crítica na prevenção da ocorrência da LP. Ao comparar custos envolvidos com prevenção e tratamento, observa-se a importância estratégica de ações preventivas, capazes de reduzir os custos com ações terapêuticas ${ }^{60}$.

O monitoramento dos fatores de risco para LP é fundamental para evitar o seu surgimento. Uma metanálise recente indicou que as atividades de prevenção da LP têm melhor relação custo/efetividade na resolução do quadro do que as atividades de tratamento. Foi verificado custo estimado do tratamento variando de US $\$ 2,500$ a US $\$ 16,000$ e a prevenção entre US $\$ 250$ e US $\$ 9,800$. Esses dados demonstram a relação dos fatores econômicos com a erradicação de fatores de risco, tornando ainda mais importante o monitoramento e o gerenciamento, desde o primeiro momento da internação hospitalar e das atividades periódicas de verificação. Outro ponto levantado foi que as intervenções nutricionais promoveram economia no tratamento em $87 \%$ a 99,99\% dos modelos elaborados por esse grupo de pesquisadores. Ainda cabe ressaltar que foi verificada tendência de melhoria na taxa de cura quando é aumentada a oferta calórico-proteica ${ }^{60}$.

As atividades de monitoramento devem ser rotineiras, incluir verificações à beira leito, desenvolvimento de protocolos e reconhecimento das populações mais suscetíveis a desenvolverem $L P$ ? .

No que se refere aos fatores de risco, eles podem ser classificados em duas categorias: fatores relacionados à exposição ao dano mecânico e à suscetibilidade e tolerância individual. Alguns fatores de risco podem ser classificados nessas duas categorias, dependendo da sua etiologia.

\section{Abordagem Prática}

O monitoramento dos fatores de risco pode acontecer em vários níveis. No que se refere à beira leito, podem ser aplicadas as escalas de verificação, como já mencionado anteriormente. Deve-se ter em mente que essas escalas não são completas no que se refere à inclusão de todos os fatores de risco e que uma avaliação complementar deve ser feita em nível individual, bem como busca ativa contínua nas atividades de monitoramento.

\section{Monitoramento de Fatores de Risco Nutricional}

O monitoramento do estado nutricional e as reavaliações nutricionais permitem verificar a aderência ao plano nutricional estabelecido na prescrição dietética e o ajuste da estimativa de necessidade de nutrientes ao longo do tempo e a condição clínica atual ${ }^{61}$.

No caso das lesões por pressão, propõe-se um pacote de medidas composto por 4 itens:

a) Rastreamento do risco nutricional;

b) Acompanhamento de disfagia e aceitação via oral ( $\mathrm{VO}$ ) (estimativa da composição das dietas e com o registro aproximado com recordatório alimentar); c) Monitorização da oferta calórico-proteica, controle de volume infundido e prescrito, em pacientes com nutrição enteral ou parenteral;

d) Uso e reposição preventiva de micronutrientes conforme necessidade clínica.

\section{Monitoramento por Indicadores de Qualidade}

Saindo do nível individual, os indicadores de qualidade podem promover um monitoramento que oferece informações sobre a gestão do cuidado recebido e as características da população alvo. Eles podem ser usados para identificar eventos adversos na atenção hospitalar, direcionar novos treinamentos à equipe assistencial, indicar novos parâmetros de monitoramento, sazonalidade de demandas ou até questões epidemiológicas ${ }^{62}$. $\bigcirc$ Quadro 6 traz exemplos de indicadores de qualidade pertinentes ao monitoramento da LP.

Quadro 6 - Sugestão de indicadores de qualidade úteis no monitoramento das lesões por pressão.

Taxa de lesão por pressão adquirida no hospital

Taxa de avaliação da pele na admissão

Taxa de avaliação de risco na admissão (Escala de Braden)

Taxa de reavaliação de risco

Índice de adesão às boas práticas

Taxa de não conformidade das medidas preventivas

Adequação da meta calórica no paciente com risco alto, muito alto e com lesão por pressão

Adequação da meta proteica no paciente com risco alto, muito alto e com lesão por pressão

Taxa de intervenção nutricional no paciente com risco alto, muito alto e com lesão por pressão

\section{AVALIAR NECESSIDADE DE NUTRIENTES ESPECÍFICOS}

\section{Referencial Teórico}

Para muitos pacientes, principalmente os desnutridos, idosos e aqueles com ingestão oral inadequada, a estratégia terapêutica é associar a dieta oral a suplementos hipercalóricos e hiperproteicos ricos em nutrientes específicos ou por meio da terapia nutricional enteral ou parenteral ${ }^{1,63}$.

As evidências demonstram que a oferta de suplemento nutricional oral (SNO), hiperproteico, rico em nutrientes específicos resulta em melhor cicatrização da LP1,64-67. O SNO hiperproteico também é eficaz na redução da incidência das $L P$, em $25 \%$ dos pacientes que apresentam risco de desenvolverem essas lesões ${ }^{68}$. Esses suplementos colaboram para a melhora do balanço positivo de nitrogênio e para todos os estágios da cicatrização'

Em uma revisão sistemática de sete estudos randomizados, Vélez-Díaz-Pallarés et al. ${ }^{69}$, com o estudo ONTOP 
(Optimal Evidence-Based Non-Drug Therapies in Older People) descreveram que a terapia nutricional enriquecida com proteína poderia levar a uma redução da ocorrência de LP em idosos quando comparado ao grupo que recebeu nutrição padrão normoproteica.

Embora a ingestão ideal de nutrientes para a cicatrização de feridas seja desconhecida, a oferta adequada de calorias, proteínas, arginina, zinco e vitaminas A, C e E melhora esse processo $1,67,70$. Alguns micronutrientes têm propriedades antioxidantes, promovem a síntese de colágeno e melhoram a resposta imunológica. Vale lembrar que, no tecido isquêmico da $L P$, grandes quantidades de radicais livres são formadas. Alguns micronutrientes, como selênio e vitaminas $A, C$ e $E$, podem reduzir a produção de radicais livres e potencialmente acelerar a cicatrização de feridas?.

Em 2017, a diretriz europeia para tratamento de pacientes crônicos com polimorbidades e LP recomendou a adição de aminoácidos específicos, como arginina, glutamina, e $\beta$-hidroxi $\beta$-metilbutirato ao SNO ou à dieta enteral, com o objetivo de acelerar o fechamento da $\mathrm{LP}^{1,71}$. Além da arginina, a prolina também tem sido estudada, por ser um importante constituinte do colágeno e essencial para cicatrização de feridas em resposta primária à lesão tecidual. Ambos apresentam relevante papel na cicatrização de feridas, pois são precursores de substratos fundamentais: oxido nítrico e colágeno ${ }^{72}$.

Uma revisão sistemática demonstrou que a dieta enteral enriquecida com arginina resultou em melhora significativa da cicatrização das lesões por pressão comparada à dieta hospitalar padrão, entre duas a doze semanas de acompanhamento ${ }^{73}$. $O$ resultado foi benéfico tanto para os pacientes desnutridos como para os não-desnutridos ${ }^{73}$. As fórmulas nutricionais orais com nutrientes específicos também melhoraram a relação custo efetividade ao correlacionar as despesas dos cuidados não nutricionais, tais como os cuidados de enfermagem e número de curativos diários, aos custos de uma suplementação nutricional específica com micronutrientes ${ }^{74}$.

Assim, a oferta da dieta hiperproteica rica em nutrientes específicos, como arginina, prolina, zinco, vitamina C, dentre outros antioxidantes, por meio da suplementação oral ou via enteral, está indicada para os pacientes com LP, pois auxiliam no seu processo de cicatrização ${ }^{72,75-80}$.

\section{Abordagem Prática}

- Está indicada dieta hiperproteica para os pacientes com risco de LP e hiperproteica, rica em nutrientes específicos, como arginina, prolina, zinco, vitamina $\mathrm{C}$ e antioxidantes, para LP instalada. Essa dieta pode ser ofertada pela via oral, na forma de suplemento nutricional oral, ou por meio da dieta enteral ${ }^{11,12,19-22,30-33,72}$;
- O SNO hiperproteico com nutrientes específicos para cicatrização está fortemente indicado para pacientes com $L P$, que não conseguem manter a ingestão oral adequada para cobrir espontaneamente os seus requerimentos nutricionais. Nestes pacientes, deve-se ofertar $\mathrm{SNO}$ ricos em nutrientes específicos para cicatrização, duas a três vezes ao dia, entre as refeições, por pelo menos quatro semanas $^{11}$;

- A suplementação de vitaminas e micronutrientes está indicada em pacientes com LP instalada, a fim de complementar o aporte nutricional caso necessário, porém deve ser avaliada individualmente pela equipe multidisciplinar de terapia nutricional (EMTN).

\section{CAPACITAR E CONSCIENTIZAR EQUIPE, FAMILIARES E PACIENTES}

\section{Referencial Teórico}

O processo do cuidar, baseado em evidências, requer dos profissionais de saúde tomadas de decisão clínicas pautadas em conhecimento científico e centralizadas na individualidade do paciente. Esse conceito vale para lesões por pressão e as ações para preveni-las e tratá-las'.

Poucos estudos abordam a relação entre a eficácia de programas educacionais de prevenção de lesões por pressão e a redução na incidência destas. Porém, demonstram: aumento nos escores médios de conhecimento sobre lesões por pressão, melhora na competência dos profissionais de saúde, no uso de instrumentos de avaliação de riscos e redução na prevalência destas lesões quando adotados programas educacionais multifacetados. Estes programas associam diversos métodos no processo de educação, principalmente os que apresentam estratégias ativas inovadoras diferentes das práticas tradicionais, tais como: apresentações didáticas, ensino à distância, programas de trainee, acesso e apoio de especialistas, debates, ensinos práticos de casos à beira leito para conscientização de profissionais da equipe multiprofissional e educação de pacientes e familiares ${ }^{81-83}$.

Outra ação fundamental para a redução na incidência de lesões por pressão é a conscientização de pacientes e familiares no seu processo de cuidar, como sujeito ativo, principal responsável e detentor do conhecimento sobre seu corpo e, por isso, fonte de controle, autonomia, valores e decisão, podendo tornar mais efetivas as práticas seguras estabelecidas pela instituição para combater as lesões, se for empoderado e orientado quanto às ações necessárias para prevenção e tratamento ${ }^{84}$.

Estudos relatam o impacto e redução de lesões após o desenvolvimento de programas de educação com pacientes e familiares, demonstrando o resultado positivo nos níveis de conhecimento de usuários e desenvolvimento de habilidades de autocuidado ${ }^{85,86}$. Torna-se importante a adequação dos 
treinamentos ao público-alvo, atentando-se à expressão verbal, nível de educação, idioma primário, deficiências sensoriais, além da escolha de diferentes métodos e atividades educacionais para envolver os indivíduos e engajá-los no processo de aprendizagem ${ }^{87}$.

\section{Abordagem Prática}

- Aplicar pesquisa de avaliação de conhecimento sobre LP com profissionais de saúde e apresentar os resultados desta para a alta gestão, objetivando desenvolvimento de iniciativas de educação direcionadas para atender às necessidades de conhecimento da equipe assistencial;

- Definir o programa de educação sobre prevenção e tratamento de LP, de acordo com a necessidade da equipe profissional e da instituição. Avaliar a eficácia e monitorar as melhorias no conhecimento, habilidades, competências e a redução na incidência de lesões por período definido;

- Definir ferramenta de avaliação específica para monitorar a Qualidade de Vida Relacionada à Saúde (QVRS) dos pacientes, conhecimento e habilidades de autocuidado. Considerar todos os aspectos físicos, psicológicos, sociais e espirituais da QVRS e suas interações ao realizar uma avaliação;

- Avaliar e considerar o conhecimento e habilidades de cuidados dos familiares e cuidadores informais para auxiliar no planejamento do cuidado. Discutir o plano de cuidados e tratamentos com eles, promovendo autogerenciamento das lesões, principalmente de indivíduos com risco contínuo;

- Determinar métodos de educação (recursos digitais, audiovisuais, educação presencial, contato telefônico) e suporte para pacientes e familiares/cuidadores.

\section{1. POR PRESSÃO}

\section{Referencial Teórico}

A documentação completa da LP é fundamental e os enfermeiros representam papel central neste processo. No entanto, se faz importante a documentação também por outros profissionais de saúde. Estudos demonstram que o registro incompleto e incorreto pode ameaçar a validade do tratamento e a qualidade da assistência prestada ${ }^{88}$.

Diante da necessidade de aprimorar a prática clínica e assistencial, proporcionando aos profissionais segurança para o processo de avaliação de lesões por pressão, diversos autores têm proposto instrumentos para mensurar feridas ${ }^{89}$. A avaliação inicial do paciente com LP deve ser abrangente e incluir histórico completo de saúde, exame físico focado no estado nutricional, dor, avaliação de risco de desenvolver novas lesões, qualidade de vida, capacidade funcional, recursos e apoios disponíveis e capacidade de adesão ao plano de prevenção e tratamento proposto. Exames laboratoriais e imagens radiológicas podem ser necessários também durante a avaliação de uma lesão90.

\section{Abordagem Prática}

A avaliação e registro da LP deve incluir':

- Local anatômico;

- Estadiamento da lesão;

- Tamanho e a área de superfície;

- Tipos de tecidos presentes e coloração;

- Condição da pele perilesional;

- Borda;

- Presença de epíbole e tunelização;

- Tipo e quantidade de exsudato;

- Presença de odor.

A avaliação da dor da ferida deve ser incluída em todas as avaliações de LP. Em pacientes com pigmentação de pele escura, priorizar a presença de calor na pele, sensibilidade aumentada e alterações na espessura tecidual perilesional ${ }^{91}$. Se a LP não demonstrar sinais de cicatrização em duas semanas, fazer reavaliação abrangente do paciente ${ }^{92}$. Esta reavaliação da lesão consiste em:

1. Avalie no início e, ao menos, uma vez por semana para monitorar a cicatrização;

2. Considere a necessidade de biópsia do leito da ferida quando a cicatrização não progredir;

3. Utilize um método uniforme e consistente para medir o tamanho da LP.

Exemplo prático hipotético de como deve ser descrita esta avaliação: paciente apresenta em região do calcâneo direito LP Estágio 4, medindo 4,5 × 4,0 cm. Em sua extensão, apresenta $40 \%$ de tecido necrótico e $60 \%$ de granulação. Região perilesional com halo de hiperemia de aproximadamente 2 $\mathrm{cm}$ da borda da lesão. Bordas irregulares e descolamento de aproximadamente $1 \mathrm{~cm}$ na posição cefalo-podálica (CFP) entre $15 \mathrm{~h}-18 \mathrm{~h}$. Ausência de tunelização. Exsudato em moderada quantidade de aspecto pio-sanguinolento e inodoro.

\section{ORIENTAR A ALTA HOSPITALAR}

\section{Referencial Teórico}

O processo educacional do paciente e de sua rede de cuidados é uma das intervenções multiprofissionais mais importante em todas as esferas de assistência à saúde. $\bigcirc$ indivíduo possui um papel valioso na prevenção de LP, assim como em todo processo de assistência que o envolve, tanto em âmbito hospitalar quanto domiciliar. Para elaboração de um plano de cuidados domiciliar é fundamental que seja avaliada a capacidade de autocuidado do indivíduo, assim como a habilidade e o conhecimento de seus cuidadores'. 
Um estudo multicêntrico randomizado com 47 pacientes desenvolveu um programa de educação voltado para prevenção de LP, com foco no autocuidado por oito semanas. grupo experimental apresentou maior disposição à adoção de comportamentos preventivos e de autocuidado, assim como aumento no conhecimento em comparação ao grupo controle. Além disso, um paciente do grupo controle apresentou uma nova LP durante o estudo ${ }^{93}$.

Estabelecer um plano de alta hospitalar estruturado com ferramentas compreensíveis para educação do paciente melhorará a capacidade da equipe multidisciplinar em garantir a efetividade do processo educacional do indivíduo ${ }^{94}$.

Diversos instrumentos podem ser utilizados no processo de educação dos pacientes e cuidadores, desde recursos e instrumentais tecnológicos, até cartilhas e manuais escritos e impressos. Cada profissional em sua realidade deve adotar o suporte que encontrar disponível e melhor se adequar ao perfil do seu público. Vale ressaltar que, em uma revisão integrativa, foi apontado que cartilhas e manuais são os recursos que mais favorecem a relação profissional/paciente, gerando impacto positivo na satisfação e no autocuidado ${ }^{95}$.

\section{Abordagem Prática}

No processo educacional do paciente sugere-se $\mathrm{s}^{50,52,96-99}$ :

- Reconhecimento das possíveis barreiras do aprendizado e comunicação: visual, auditiva, fala, outras (cultural, religiosa, psicomotora, emocional);

- Identificação da pessoa envolvida no processo educacional para engajamento e empoderamento (paciente, parente, cuidador ou equipe de home care);

- Iniciar o processo de orientação durante o período de hospitalização e o mais precocemente possível;

- Definir o melhor método de ensino de acordo com o nível de entendimento do paciente e dos envolvidos: demonstração, audiovisual, verbal, folheto;

- Avaliar a compreensão do indivíduo orientado por meio da verbalização, recusa, capacidade de demonstração ou não entendimento;

- Identificar a necessidade de reforço das orientações;

- Detectar se o objetivo foi atingido ou não, realizando o teach back (confirmação se houve o entendimento, solicitando que o envolvido explique o que foi orientado) para avaliar a compreensão sobre as instruções de alta e a capacidade de realizar o autocuidado.

Considerando um plano de cuidados domiciliar multidisciplinar para um indivíduo em vigência de uma $L P$, este deve conter os seguintes tópicos:

- Etiologia das lesões por pressão, incluindo a anatomia da pele e fatores de risco para o desenvolvimento;
- Aconselhamento dietético;

- Recomendações para hábitos saudáveis;

- Abordagem com relação ao tabagismo e consumo de álcool;

- Higiene pessoal;

- Estratégias de redistribuição de pressão ao sentar-se e deitar-se;

- Uso de colchões e almofadas adequados;

- Uso de roupas e calçados adequados;

- Inspeção e cuidados com a pele;

- Tratamento de feridas;

- Plano de exercício de acordo com a funcionalidade do indivíduo.

É de extrema importância que a equipe multiprofissional suporte a continuidade de tratamento por meio de teleconsulta, ambulatório ou visita domiciliar.

\section{CONCLUSÃO}

A integração da equipe interdisciplinar na prevenção e no tratamento da LP, com foco na qualidade, segurança e experiência do paciente, é de grande importância. Para tal, fomentar os profissionais da saúde a mitigar os riscos por meio da sensibilização sobre essa lesão (evitável), que afeta diretamente a qualidade de vida dos pacientes, além dos impactos econômicos para o sistema da saúde, se faz necessário. Dessa maneira, a sequência dos 12 passos estabelecidos nesta campanha é sugerida como medida para auxiliar os profissionais da saúde neste manejo.

\section{REFERENCIAS}

1. National Pressure Ulcer Advisory Panel, European Pressure Ulcer Advisory Panel and Pan Pacific Pressure Injury Alliance. Prevention and treatment of pressure ulcers: quick reference guide. Osborne Park: Cambridge Media; 2019.

2. Murphree RW. Impairments in skin integrity. Nurs Clin North Am. 2017;52(3):405-17.

3. Agência Nacional de Vigilância Sanitária (Anvisa). Relatório nacional de incidentes relacionados à assistência à saúde. Boletim Segurança do Paciente e Qualidade em Serviços de Saúde. [cited 2020 Feb 17]. Available from: https://www20.anvisa.gov.br/segurancadopaciente/index. $\mathrm{php} /$ publicacoes/category/relatorios-dos-estados

4. Gorecki C, Brown JM, Nelson EA, Briggs M, Schoonhoven L, Dealey C, et al; European Quality of Life Pressure Ulcer Project Group. Impact of pressure ulcers on quality of life in older patients: a systematic review. J Am Geriatr Soc. 2009;57(7):1175-83.

5. Gorecki C, Nixon J, Madill A, Firth J, Brown JM. What influences the impact of pressure ulcers on health-related quality of life? A qualitative patient-focused exploration of contributory factors. J Tissue Viability. 2012;21(1):3-12.

6. Dreyfus J, Gayle J, Trueman P, Delhougne G, Siddiqui A. Assessment of risk factors associated with hospital-acquired pressure injuries and impact on health care utilization and cost outcomes in US hospitals. Am J Med Qual. 2018;33(4):348-58.

7. Souza MFC, Zanei SSV, Whitaker IY. Risk of pressure injury in the ICU: transcultural adaptation and reliability of EVARUCI. Acta Paul Enferm. 2018;31(2):201-8. 
8. Braden B, Bergstrom N. A conceptual schema for study of the etiology of pressure sores. Rehabil Nurs. 1987;12(1):8-12.

9. Chiari P, Forni C, Guberti M, Gazineo D, Ronzoni S, D'Alessandro F. Predictive factors for pressure ulcers in an older adult population hospitalized for hip fractures: a prognostic cohort study. PLoS One. 2017;12(1):e0169909.

10. Ranzani OT, Simpson ES, Japiassú AM, Noritomi DT; Amil Critical Care Group. The challenge of predicting pressure ulcers in critically ill patients. a multicenter cohort study. Ann Am Thorac Soc. 2016;13(10):1775-83.

11. Nassaji M, Askari Z, Ghorbani R. Cigarette smoking and risk of pressure ulcer in adult intensive care unit patients. Int J Nurs Pract. 2014;20(4):418-23.

12. Ham HW, Schoonhoven LL, Schuurmans MM, Leenen LL. Pressure ulcer development in trauma patients with suspected spinal injury: the influence of risk factors presents in the emergency department. Int Emerg Nurs. 2017;30:13-9.

13. Sternal D, Wilczyński K, Szewieczek J. Pressure ulcers in palliative ward patients: hyponatremia and low blood pressure as indicators of risk. Clin Interv Aging. 2017;12:37-44.

14. Cox J, Roche S. Vasopressors and development of pressure ulcers in adult critical care patients. Am J Crit Care. 2015;24(6):501-10.

15. Bly D, Schallom M, Sona C, Klinkenberg D. A model of pressure, oxygenation, and perfusion risk factors for pressure ulcers in the intensive care unit. Am J Crit Care. 2016;25(2):156-64.

16. Joseph C, Nilsson Wikmar L. Prevalence of secondary medical complications and risk factors for pressure ulcers after traumatic spinal cord injury during acute care in South Africa. Spinal Cord. 2016;54(7):535-9.

17. Brienza D, Krishnan S, Karg P, Sowa G, Allegretti AL. Predictors of pressure ulcer incidence following traumatic spinal cord injury: a secondary analysis of a prospective longitudinal study. Spinal Cord. 2018;56(1):28-34.

18. Chaboyer WP, Thalib L, Harbeck EL, Coyer FM, Blot S, Bull CF, et al. Incidence and prevalence of pressure injuries in adult intensive care patients: a systematic review and meta-analysis. Crit Care Med. 2018;46(11):e1074-81.

19. Tayyib N, Coyer F, Lewis P. Saudi Arabian adult intensive care unit pressure ulcer incidence and risk factors: a prospective cohort study. Int Wound J. 2016;13(5):912-9.

20. Forni C, D'Alessandro F, Genco R, Mini S, Notarnicola T, Vitulli A, et al. Prospective prognostic cohort study of pressure injuries in older adult patients with hip fractures. Adv Skin Wound Care. 2018;31(5):218-24.

21. Australian Wound Management Association. Pan Pacific clinical practice guideline for the prevention and management of pressure injury. Osborne Park: Cambridge Media; 2012.

22. Governo do Distrito Federal. Secretaria de Estado de Saúde. Protocolo de Atenção à Saúde. Segurança do paciente: prevenção de lesão por pressão (LP). [cited $2020 \mathrm{Feb}$ 17]. Available from: http://www.saude.df.gov.br/ wp-conteudo/uploads/2018/04/Seguranca-do-Paciente-prevencao-deLesao-por-Pressao-LP-2.pdf

23. Holloway S, Jones $\mathrm{V}$. The importance of skin care and assessment. Br J Nurs. 2005;14(22):1172-6.

24. Bateman SD, Roberts S. Moisture lesions and associated pressure ulcers: getting the dressing regimen right. Wounds UK, 2013;9(2):97-102.

25. Associação Brasileira de Estomaterapia e Associação Brasileira de Enfermagem em Dermatologia. Classificação das lesões por pressão Consenso NPUAP 2016 - Adaptada Culturalmente para o Brasil. 2016. [cited 2019 Dec 9]. Available from: http://www.sobest.org.br/textod/35

26. Edsberg LE, Black JM, Goldberg M, McNichol L, Moore L, Sieggreen M. Revised National Pressure Ulcer Advisory Panel Pressure Injury Staging System: revised pressure injury staging system. J Wound Ostomy Continence Nurs. 2016;43(6):585-97.

27. Ilizaka S, Okuwa M, Sugama J, Sanada H. The impact of malnutrition and nutrition-related factors on the development and severity of pressure ulcers in older patients receiving home care. Clin Nutr. 2010;29(1):47-53.

28. Eglseer D, Hödl M, Lohrmann C. Nutritional management of older hospitalised patients with pressure injuries. Int Wound J. 2019;16(1):226-32.

29. Lyder CH, Preston J, Grady JN, Scinto J, Allman R, Bergstrom N, et al. Quality of care for hospitalized Medicare patients at risk for pressure ulcers. Arch Intern Med. 2001;161(12):1549-54.

30. Skipper A, Ferguson M, Thompson K, Castellanos VH, Porcari J. Nutrition screening tools: an analysis of the evidence. JPEN J Parenter Enteral Nutr. 2012;36(3):292-8.

31. National Institute for Health and Care Excellence. Pressure ulcers: prevention and management. London: NICE; 2014.
32. Wound, Ostomy and Continence Nurses Society-Wound Guidelines Task Force. WOCN 2016 guideline for prevention and management of pressure injuries (ulcers): an executive summary. J Wound Ostomy Continence Nurs. 2017;44(3):241-6.

33. Registered Nurses'Association of Ontario. Assessment and management of pressure injuries for the interprofessional team. $3^{\text {rd }}$ ed. Toronto: Registered Nurses' Association of Ontario; 2016.

34. Nestle Nutrition Institute. Mini nutrition assessment MNA®. [cited 2019 May 8]. Available from: https://www.mnaelderly.com/forms/mini/ mna_mini_english.pdf

35. Yatāe MS , Taguchi F, Ishida, Sato A, Kameda T, Ueno S, et al. Mini nutritional assessment as a useful method of predicting the development of pressure ulcers in elderly inpatients. J Am Geriatr Soc. 2013;61(10):1698-704.

36. Meehan A, Loose C, Bell J, Partridge J, Nelson J, Goates S. Health system quality improvement: impact of prompt nutrition care on patient outcomes and health care costs. J Nurs Care Qual. 2016;31(3):217-23.

37. Horn SD, Bender SA, Ferguson ML, Smout RJ, Bergstrom N, Taler G, et al. The National Pressure Ulcer Long-Term Care Study: pressure ulcer development in long-term care residents. J Am Geriatr Soc. 2004;52(3):359-67.

38. Gonzalez EDL, Mendivil LLL, Garza DPS, Hermosillo HG, Chavez JHM, Corona RP. Low handgrip strength is associated with a higher incidence of pressure ulcers in hip fractured patients. Acta Orthop Belg. 2018;84(3):284-91.

39. Sancho A, Albiol R, Mach N. Relationship between nutritional status and the risk of having pressure ulcers in patients included in a home care program. Aten Primaria. 2012;44(10):586-94.

40. Dalapicola MM. A importância do suporte nutricional em pacientes portadores de úlcera de pressão. Cad Saúde Desenv. 2013;2(2):76-89.

41. Little MO. Nutrition and skin ulcers. Curr Opin Clin Nutr Metab Care. 2013;16(1):39-49.

42. Campos AC, Groth AK, Branco AB. Assessment and nutritional aspects of wound healing. Curr Opin Clin Nutr Metab Care. 2008;11(3):281-8.

43. Campos ACL, Branco AB, Groth AK. Cicatrização de feridas. Arq Bras Cir Dig. 2007;20(1):51-8.

44. National Health and Medical Research Council, Australian Government Department of Health and Ageing, New Zealand Ministry of Health, Nutrient Reference Values for Australia and New Zealand. Canberra: National Health and Medical Research Council; 2017.

45. Volkert D, Beck AM, Cederholm T, Cruz-Jentoft A, Goisser S, Hooper L, et al. ESPEN guideline on clinical nutrition and hydration in geriatrics. Clin Nutr. 2019;38(1):10-47.

46. McClave SA, Taylor BE, Martindale RG, Warren MM, Johnson DR, Braunschweig C, et al; Society of Critical Care Medicine; American Society for Parenteral and Enteral Nutrition. Guidelines for the provision and assessment of nutrition support therapy in the adult critically ill patient: Society of Critical Care Medicine (SCCM) and American Society for Parenteral and Enteral Nutrition (A.S.P.E.N.). JPEN J Parenter Enteral Nutr. 2016;40(2):159-211.

47. Mueller CM. The ASPEN Adult Nutrition Support Core Curriculum. Vol. USA 2017 American Society for Enteral and Parenteral Nutrition.

48. Alvarez OM, Meehan M, Ennis W, Thomas DR, Ferris FD, Kennedy KL, et al. Chronic wounds: palliative management for the frail population. Wounds. 2002;14(8):4S-27s.

49. Wu G, Bazer FW, Burghardt RC, Johnson GA, Kim SW, Knabe DA, et al. Proline and hydroxyproline metabolism: implications for animal and human nutrition. Amino Acids. 2011;40(4):1053-63.

50. Piovacari SMF, Toledo DO, Figueiredo EJA. Equipe multiprofissional de terapia nutricional: EMTN em prática. São Paulo: Atheneu; 2017.

51. Castro MG, Ribeiro PC, Souza IAO, Cunha HFR, Silva MHN, Rocha EEM, et al. Diretriz brasileira de terapia nutricional no paciente grave. BRASPEN J. 2018;33(Supl 1):2-36.

52. Toledo DO, Piovacari SMF, Horie LM, Matos LBN, Castro MG, Ceniccola GD, et al. Campanha "Diga não à desnutrição": 11 passos importantes para combater a desnutrição hospitalar. BRASPEN J. 2018;33(1):86-100.

53. Chew HSJ, Thiara E, Lopez V, Shorey S. Turning frequency in adult bedridden patients to prevent hospital-acquired pressure ulcer: a scoping review. Int Wound J. 2018;15(2):225-36.

54. Institute for Healthcare Improvement. How-to-guide: prevent pressure ulcers. Cambridge: Institute for Healthcare Improvement; 2011. [cited 2020 Jan 20]. Available from: http://www.ihi.org

55. Brindle CT, Malhotra R, O’Rourke S, Currie L, Chadwik D, Falls P, et al. Turning and repositioning the critically ill patient with hemodynamic instability: a literature review and consensus recommendations. J Wound Ostomy Continece Nurs. 2013;40(3):254-67. 
56. Smit I, Harrison L, Letzkus L, Quatrara B. What factors are associated with the development of pressure ulcers in a medical intensive care unit? Dimens Crit Care Nur. 2016;35(1):37-41.

57. Castro M, Pompilio CE. Protocolos de terapia nutricional em unidades de terapia intensiva. In: Toledo D, Castro M, eds. Terapia nutricional em UTI. Rio de Janeiro: Rubio; 2015.

58. Associação Brasileira de Nutrição. Manual orientativo. Sistematização no cuidado de nutrição. São Paulo: Associação Brasileira de Nutrição; 2014.

59. Gonçalves TJ, Horie LM, Gonçalves SEAB, Bacchi MK, Bailer MC, Barbosa-Silva TG, et al. Diretriz BRASPEN de terapia nutricional no envelhecimento. BRASPEN J. 2019;34(supl 3):2-58.

60. Wong A, Goh G, Banks MD, Bauer JD. Economic evaluation of nutrition support in the prevention and treatment of pressure ulcers in acute and chronic care settings: a systematic review. JPEN J Parenter Enteral Nutr. 2019;43(3):376-400

61. Alvite MFL, Piovacari SMF, Nascimento DBD. Parecer da BRASPEN sobre prescrição de volume, consistência e suplemento nutricional no paciente disfágico. BRASPEN J. 2019;34(4):418-20.

62. Verotti CCG, Ceniccola GD, Rajendram R. Top ten quality indicators for nutritional therapy. Diet and Nutrition in Critical Care. 2015;417-28.

63. Blanc G, Meier MJ, Stocco JG, Roehrs H, Crozeta K, Barbosa DA. Effectiveness of enteral nutritional therapy in the healing process of pressure ulcers: a systematic review. Rev Esc Enferm USP. 2015;49(1):152-61.

64. Allen B. Effects of a comprehensive nutritional program on pressure ulcer healing, length of hospital stay, and charges to patients. Clin Nurs Res. 2013;22(2):186-205

65. Royal College of Nursing (UK). The management of pressure ulcers in primary and secondary care: a clinical practice guideline [Internet]. London: Royal College of Nursing (UK); 2005.

66. Bourdel-Marchasson I, Barateau M, Rondeau V, Dequae-Merchadou L, Salles-Montaudon N, Emeriau JP, et al. A multi-center trial of the effects of oral nutritional supplementation in critically ill older in patients. GAGE Group. Groupe Aquitain Geriatrique d'Evaluation. Nutrition. 2000;16(1):1-5.

67. Ek AC, Unosson M, Larsson J, Von Schenck H, Bjurulf P. The development and healing of pressure sores related to the nutritional state. Clin Nutr. 1991;10(5):245-50.

68. Raffoul W, Far MS, Cayeux MC, Berger MM. Nutritional status and food intake in nine patients with chronic low-limb ulcers and pressure ulcers: importance of oral supplements. Nutrition. 2006;22(1):82-8.

69. Vélez-Díaz-Pallarés M, Lozano-Montoya I, Abraha I, Cherubini A, Soiza RL, O'Mahony D, et al. Nonpharmacologic interventions to heal pressure ulcers in older patients: an overview of systematic reviews (The SENATOR-ONTOP Series). J Am Med Dir Assoc. 2015;16(6):448-69.

70. Saghaleini SH, Dehghan K, Shadvar K, Sanaie S, Mahmoodpoor A, Ostadi Z. Pressure ulcer and nutrition. Indian J Crit Care Med. $2018 ; 22(4): 283-9$

71. Gomes F, Schuetz P, Bounoure L, Austin P, Ballesteros-Pomar M, Cederholm $\mathrm{T}$, et al. ESPEN guidelines on nutritional support for polymorbid internal medicine patients. Clin Nutr. 2018;37(1):336-53.

72. Albaugh VL, Mukherjee K, Barbul A. Proline precursors and collagen synthesis: biochemical challenges of nutrient supplementation and wound healing. J Nutr. 2017;147(11):2011-7.

73. Liu P, Shen WQ, Chen HL. Efficacy of arginine-enriched enteral formulas for the healing of pressure ulcers: a systematic review. J Wound Care. 2017;26(6):319-23.

74. Cereda E, Klersy C, Andreola M, Pisati R, Schols JM, Caccialanza R, et al; OligoElement Sore Trial (OEST) Study Group. Cost-effectiveness of a disease-specific oral nutritional support for pressure ulcer healing. Clin Nutr. 2017;36(1):246-52.

75. Banks MD, Ross LJ, Webster J, Mudge A, Stankiewicz M, Dwyer K, et al. Pressure ulcer healing with an intensive nutrition intervention in an acute setting: a pilot randomised controlled trial. J Wound Care. 2016;25(7):384-92.

76. Desneves KJ, Todorovic BE, CassarA, Crowe TC. Treatment with supplementary arginine, vitamin $\mathrm{C}$ and zinc in patients with pressure ulcers: a randomized controlled trial. Clin Nutr. 2005;24(6):979-87.

77. Cereda E, Gini A, Pedrolli C, VanottiA. Disease-specific, versus standard, nutritional support for the treatment of pressure ulcers in institutionalized older adults: a randomized controlled trial. J Am Geriatr Soc. 2009;57(8):1395-402.

78. Brewer S, Desneves K, Pearce L, Mills K, Dunn L, Brown D, et al. Effect of an arginine-containing nutritional supplement on pressure ulcer healing in community spinal patients. J Wound Care. 2010;19(7):311-6.
79. Leigh B, Desneves K, Rafferty J, Pearce L, King S, Woodward MC, et al. The effect of different doses of an arginine-containing supplement on the healing of pressure ulcers. J Wound Care. 2012;21(3):150-6.

80. Frías Soriano L, Lage Vázquez MA, Maristany CP, Xandri Graupera JM, Wouters-Wesseling W, Wagenaar L. The effectiveness of oral nutritional supplementation in the healing of pressure ulcers. J Wound Care. 2004;13(8):319-22

81. Feng H, Li G, Xu C, Ju C. Educational campaign to increase knowledge of pressure ulcers. Br J Nurs. 2016;25(12):S30-5.

82. Kwong EWY,LauATY, Lee RLP, Kwan RYC. Apressure ulcer prevention programme specially designed for nursing homes: does it work? J Clin Nurs. 2011;20(19-20):2777-86.

83. Ilesanmi RE, Oluwatosin OM. A quasi-experimental study to assess an interactive educational intervention on nurse' knowledge of pressure ulcer prevention in Nigeria. Ostomy Wound Manage. 2016;62(4):30-40.

84. Viana I, Aguiar FC, Rios SO, Mendes VLPS, Garcia EG. Direitos do paciente, comunicação e a obrigação de informar. Rev Baiana Saúde Pública. 2016;40(supl 1):182-201.

85. Rintala DH, Garber SL, Friedman JD, Holmes SA. Preventing recurrent pressure ulcers in veterans with spinal cord injury: impact of a structured education and follow-up intervention. Arch Phys Med Rehabil. 2008;89(8):1429-41

86. Garber SL, Rintala DH, Holmes SA, Rodriguez GP, Friedman J. A structured educational model to improve pressure ulcer prevention knowledge in veterans with spinal cord dysfunction. J Rehabil Res Dev. 2002:39(5):575-88

87. Chaboyer W, Harbeck E, Bucknall T, McInnes E, Thalib L, Whitty J, et al. Initial psychometric testing and validation of the patient participation in pressure injury prevention scale. J Adv Nurs. 2017; 73(9):2237-47.

88. Hart S, Bergquist S, Gajewski B, Dunton N. Reliability testing of the National Database of Nursing Quality Indicators pressure ulcer indicator. J Nurs Care Qual. 2006;21(3):256-65.

89. Gabison S, McGillivray C, Hitzig SL, Nussbaum E. A study of the utility and equivalency of 2 methods of wound measurement: digitized tracing versus digital photography. Adv Skin Wound Care. 2015;28(6):252-8.

90. Langemo D, Spahn J, Spahn T, Pinnamaneni VC. Comparison of standardized clinical evaluation of wounds using ruler length by width and Scout length by width measure and Scout perimeter trace. Adv Skin Wound Care. 2015;28(3):116-21.

91. Bliss DZ, Gurvich O, Savik K, Eberly LE, Harms S, Mueller C, et al. Racial and ethnic disparities in the healing of pressure ulcers present at nursing home admission. Arch Gerontol Geriatr. 2017;72:187-94.

92. Choi EP, Chin WY, Wan EY, Lam CL. Evaluation of the internal and external responsiveness of the Pressure Ulcer Scale for Healing (PUSH) tool for assessing acute and chronic wounds. J Adv Nurs. 2016;72(5):1134-43

93. Kim JY, Cho E. Evaluation of a self-efficacy enhancement program to prevent pressure ulcers in patients with a spinal cord injury. Jpn J Nurs Sci. 2017;14(1):76-86.

94. Seyedin H, Goharinezhad S, Vatankhah S, Azmal M. Patient education process in teaching hospitals of Tehran University of Medical Sciences. Med J Islam Repub Iran. 2015;29:220.

95. Koerich C, Lanzoni GMM, Coimbra R, Tavares KS, Erdmann AL. Recursos e competências para gestão de práticas educativas por enfermeiros: revisão integrativa. Rev Gaúcha Enferm. 2019;40:e20180031.

96. Weintraub B, Jensen K, Colby K. Improving hospital wide patient flow at Northwest Community Hospital. In: Joint Commission Resources. Managing patient flow in hospitals: strategies and solutions. 2nd ed. Springfield: JCR Department of Publications; 2010. p.129-52.

97. Rutherford P, Nielsen GA, Taylor J, Bradke P, Coleman E. How to guide: improving transitions from the hospital to community settings to reduce avoidable rehospitalizations. Cambridge: Institute for Healthcare Improvement; 2013

98. Ryckman FC, Adler MD, Anneken AM, Bedinghaus CA, Clayton PJ, Hays KR, et al. Cincinnati Children's Hospital Medical Center: redesigning perioperative flow using operations management tools to improve access and safety. In: Joint Commission Resources. Managing patient flow in hospitals: strategies and solutions. $2^{\text {nd }}$ ed. Springfield: JCR Department of Publications; 2010. p.96-112.

99. Hoyer EH, Brotman DJ, Apfel A, Leung C, Boonyasai RT, Richardson $\mathrm{M}$, et al. Improving outcomes after hospitalization: a prospective observational multicenter evaluation of care coordination strategies for reducing 30-day readmissions to Maryland hospitals. J Gen Intern Med. 2018;33(5):621-7. 